\title{
O)
}

\section{AIAA 98-3107}

Experimental, Theoretical, and Computational Investigation of Separated Nozzle Flows

C.A. Hunter

NASA Langley Research Center

Hampton, Virginia

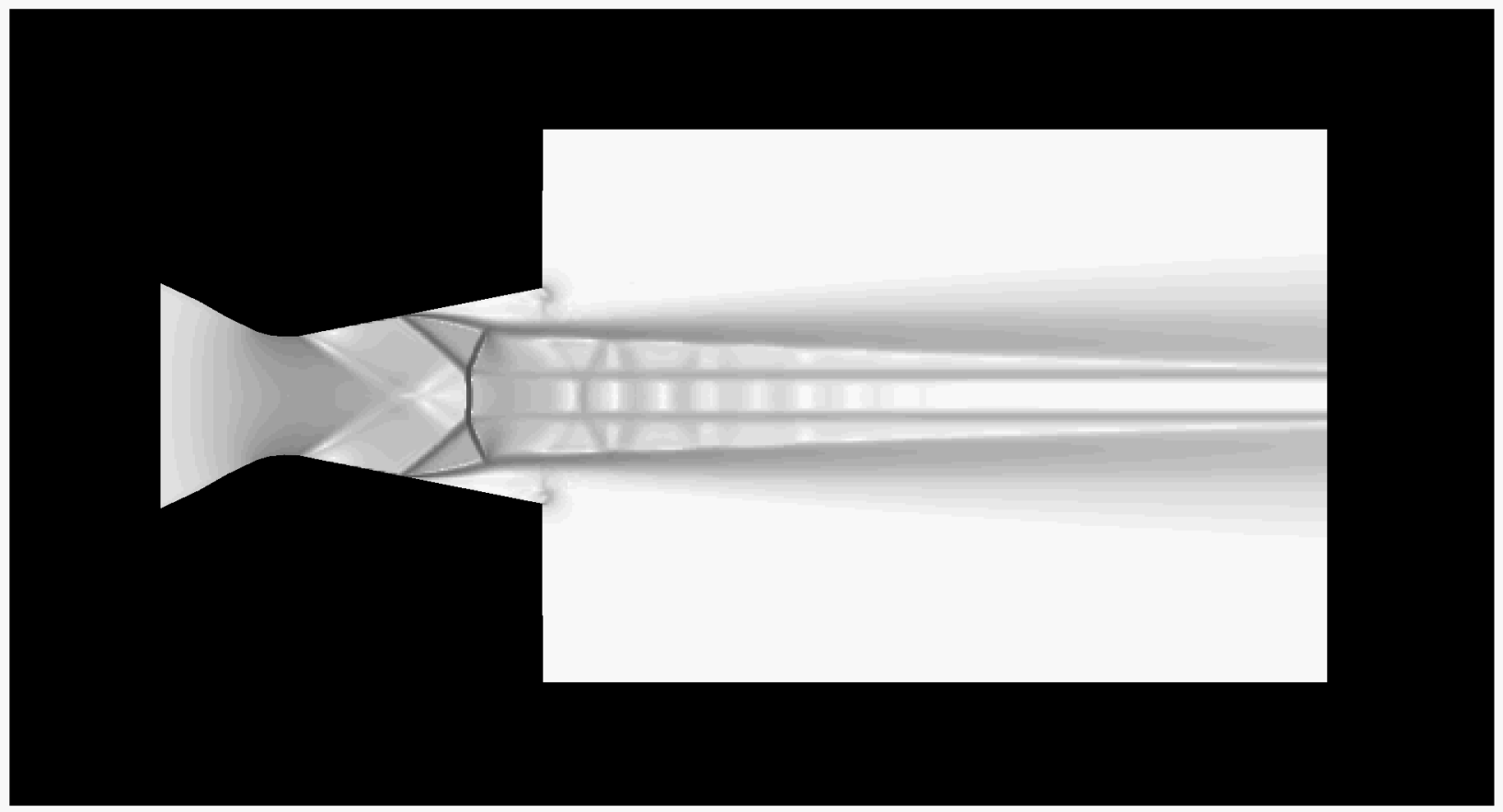

\section{4th AIAA/ASME/SAE/ASEE Joint Propulsion Conference \& Exhibit July 13-15, 1998 / Cleveland, $\mathrm{OH}$}




\title{
EXPERIMENTAL, THEORETICAL, AND COMPUTATIONAL INVESTIGATION OF SEPARATED NOZZLE FLOWS
}

\author{
Craig A. Hunter * \\ NASA Langley Research Center \\ Hampton, Virginia
}

\begin{abstract}
A detailed experimental, theoretical, and computational study of separated nozzle flows has been conducted. Experimental testing was performed at the NASA Langley 16-Foot Transonic Tunnel Complex. As part of a comprehensive static performance investigation, force, moment, and pressure measurements were made and schlieren flow visualization was obtained for a subscale, non-axisymmetric, two-dimensional, convergentdivergent nozzle. In addition, two-dimensional numerical simulations were run using the computational fluid dynamics code PAB3D with two-equation turbulence closure and algebraic Reynolds stress modeling. For reference, experimental and computational results were compared with theoretical predictions based on one-dimensional gas dynamics and an approximate integral momentum boundary layer method.
\end{abstract}

Experimental results from this study indicate that offdesign overexpanded nozzle flow was dominated by shock induced boundary layer separation, which was divided into two distinct flow regimes; threedimensional separation with partial reattachment, and fully detached two-dimensional separation. The test nozzle was observed to go through a marked transition in passing from one regime to the other. In all cases, separation provided a significant increase in static thrust efficiency compared to the ideal prediction. Results indicate that with controlled separation, the entire overexpanded range of nozzle performance would be within $10 \%$ of the peak thrust efficiency. By offering savings in weight and complexity over a conventional mechanical exhaust system, this may allow a fixed geometry nozzle to cover an entire flight envelope.

The computational simulation was in excellent agreement with experimental data over most of the test range, and did a good job of modeling internal flow and thrust performance. An exception occurred at low nozzle pressure ratios, where the two-dimensional computational model was inconsistent with the threedimensional separation observed in the experiment. In

* Aerospace Engineer, Configuration Aerodynamics Branch, Aero- and Gas-Dynamics Division. Member AIAA

Copyright $\odot 1998$ by the American Institute of Aeronautics and Astronautics, Inc. No copynght is asserte The U.S. Government government purposes. All other rights are reserved by the copyright owner. general, the computation captured the physics of the shock - boundary layer interaction and shock induced boundary layer separation in the nozzle, though there were some differences in shock structure compared to experiment. Though minor, these differences could be important for studies involving flow control or thrust vectoring of separated nozzles. Combined with other observations, this indicates that more detailed, threedimensional computational modeling needs to be conducted to more realistically simulate shockseparated nozzle flows.

\section{Introduction}

In the world of modern fluid dynamics, boundary layer separation is typically regarded as a "tragic" phenomena, and rightly so; performance penalties and instabilities often accompany separation, and these can have a detrimental or catastrophic effect on almost any fluid dynamic system. Putting this aside, however, it is important to realize that boundary layer separation is a natural process; it is the means by which a viscous flow adjusts to its surroundings under particular conditions. In certain cases, this mechanism can have many benefits, ranging from enhanced performance to flow control

Consider an overexpanded convergent-divergent (CD) nozzle. This condition occurs because the nozzle expansion ratio is too large for a given nozzle pressure ratio (NPR). Supersonic nozzle flow is driven to expand below exit back pressure, and a nonisentropic, discontinuous shock jump is required to adjust. Ideally, flow stays attached downstream of the shock, but in reality it usually separates from the nozzle flap if the expansion ratio is high enough or the flap angle steep enough. An illustration of flow in a shock separated nozzle is given in figure 1 .

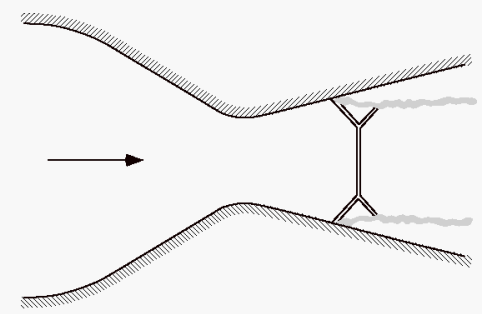

Figure 1: Overexpanded CD Nozzle with Separation 
At first glance, it is often surprising that nozzle separation is accompanied by an increase in thrust efficiency over the attached case ${ }^{1}$, but it shouldn't be. A major effect of boundary layer separation is that it redefines the "effective" geometry of a fluid dynamic system by displacing the inviscid flow. In a CD nozzle, separation moves the jet detachment point upstream, causing a change in the effective nozzle geometry to one that is shorter and has a lower expansion ratio. For a given NPR, this alleviates overexpansion and improves thrust efficiency. By acting like a natural "adjustment mechanism", separation allows an overexpanded nozzle flow to reach a more efficient thermodynamic balance.

Earlier studies by Asbury, et. al. ${ }^{2}$ and Hunter ${ }^{3}$ showed that off-design nozzle thrust efficiency could be improved beyond what occurs naturally by encouraging stable separation (with a passive porous cavity) and controlling the location and extent of that separation. In addition, this work demonstrated that effective thrust vectoring could be attained by using asymmetric shock - boundary layer interaction control. The recent growth in micro adaptive flow control technology ${ }^{4}$ and active flow control concepts offers further means by which to control and stabilize a separated nozzle flow for performance enhancement or thrust vectoring.

Many of these new and emerging active and passive flow control technologies remain unexplored in exhaust systems. Current aircraft typically use mechanical systems for nozzle area control and thrust vectoring. While effective, these systems can be heavy, complex, expensive to maintain, and prone to fatigue and failure. They are also difficult to integrate aerodynamically and can be a primary source of drag. With the addition of other requirements such as IR suppression, noise suppression, and low-observable shaping, the design and integration of an efficient mechanical exhaust system becomes all the more challenging.

The capabilities of future aircraft will depend on the development of simple, lightweight exhaust systems that are aerodynamically efficient, compact, and inexpensive. In light of this, there is tremendous potential to improve aircraft system performance by replacing mechanical nozzle systems with efficient fixed geometry configurations that use separation to enhance and control off-design performance. For this reason, and because of the complicated relationship between overexpansion, shock induced separation, and thrust efficiency, an understanding of separated nozzle flows is critical from experimental, theoretical, and computational standpoints. This paper addresses that understanding.

\section{Nomenclature}

\begin{tabular}{|c|c|}
\hline$A_{e}$ & Nozzle Exit Area, in ${ }^{2}$ \\
\hline$A_{t}$ & Nozzle Throat Area, in ${ }^{2}$ \\
\hline$A_{e} / A_{t}$ & Nozzle Expansion Ratio \\
\hline $\mathrm{BL}$ & Boundary Layer \\
\hline $\mathrm{F}$ & Axial Thrust. lb \\
\hline $\mathrm{F}_{1}$ & Ideal Isentropic Thrust, lb \\
\hline $\mathrm{F} / \mathrm{F}_{\mathrm{i}}$ & Thrust Efficiency Ratio \\
\hline $\mathrm{h}$ & BL Wall Normal Coordinate, $\mathrm{ft}$ \\
\hline $\mathrm{h}^{+}$ & BL Normal in Wall Variables, $\mathrm{h}^{+}=\mathrm{hu}^{*} / \mathrm{N}$ \\
\hline $\mathrm{k}$ & TKE per Unit Mass, $\mathrm{ft}^{2} / \mathrm{s}^{2}$ \\
\hline $\mathrm{k}_{\mathrm{PK}}$ & Peak $\mathrm{k}$ in the Boundary Layer, $\mathrm{ft}^{2} / \mathrm{s}^{2}$ \\
\hline $\mathrm{k}_{\infty}$ & Computational Lower Limit for $\mathrm{k}, \mathrm{ft}^{2} / \mathrm{s}^{2}$ \\
\hline$\ell$ & Boundary Layer Streamwise Coordinate, $\mathrm{ft}$ \\
\hline M & Local Mach Number \\
\hline$M_{a}$ & Ambient Freestream Mach Number \\
\hline $\mathrm{M}_{\mathrm{s}}$ & Undisturbed Separation Mach Number \\
\hline $\mathrm{M}_{1}$ & Mach Number Upstream of a Shock \\
\hline NPR & Nozzle Pressure Ratio, $N P R=p_{o j} / p_{a}$ \\
\hline $\mathrm{p}$ & Static Pressure, psi \\
\hline $\mathrm{p}_{\mathrm{a}}$ & Ambient Pressure, psi \\
\hline $\mathrm{p}_{\mathrm{s}}$ & Undisturbed Separation Pressure, psi \\
\hline $\mathrm{p}_{\mathrm{oj}}$ & Jet Total Pressure \\
\hline $\mathrm{Re}_{\ell}$ & Reynolds Number Based on $\ell, \operatorname{Re}_{\ell}=\mathrm{u}_{\ell} \ell / v_{\mathrm{w}}$ \\
\hline $\operatorname{Re}_{\delta}$ & Reynolds Number Based on $\delta, \operatorname{Re}_{\delta}=u_{e} \delta / v_{w}$ \\
\hline $\mathrm{t}$ & time, $\mathrm{s}$ \\
\hline $\mathrm{T}_{\mathrm{a}}$ & Ambient Temperature, ${ }^{\circ} \mathrm{R}$ \\
\hline $\mathrm{T}_{\mathrm{oj}}$ & Jet Stagnation Temperature, ${ }^{\circ} \mathrm{R}$ \\
\hline TKE & Turbulent Kinetic Energy, $\mathrm{lb} / \mathrm{ft}^{2}$ \\
\hline $\mathrm{u}$ & BL Velocity Parallel to Wall, $\mathrm{ft} / \mathrm{s}$ \\
\hline $\mathrm{u}_{\mathrm{e}}$ & BL Edge Velocity, $\mathrm{ft} / \mathrm{s}$ \\
\hline $\mathrm{u}^{*}$ & BL Friction Velocity, $\mathbf{u}^{*^{2}}=v_{\mathrm{w}}(\partial \mathrm{u} / \partial \mathrm{h})_{\mathrm{h}=0}$ \\
\hline $\mathrm{u}^{+}$ & BL Velocity in Wall Variables, $\mathbf{u}^{+}=\mathbf{u} / \mathbf{u}^{*}$ \\
\hline & ith Component of Mean Flow Velocity, ft/s \\
\hline$\left\langle u_{i}^{\prime} u_{j}^{\prime}\right\rangle$ & Reynolds Stress Tensor, $\mathrm{ft}^{2} / \mathrm{s}^{2}$ \\
\hline $\mathrm{x}$ & Streamwise Coordinate, in \\
\hline $\mathbf{x}_{\mathrm{s}}$ & Streamwise Location of Nozzle Shock, in \\
\hline $\mathrm{x}_{\mathrm{t}}$ & Streamwise Location of Nozzle Throat, in \\
\hline $\mathrm{x}_{\mathrm{i}}$ & ith Spatial Coordinate, $\mathrm{ft}$ \\
\hline $\mathrm{y}$ & Streamwise Normal (Vertical) Coordinate, in \\
\hline$\alpha$ & Flow Angle, Clockwise from Horizontal \\
\hline$\beta$ & Oblique Shock Inclination Angle, degrees \\
\hline$\delta_{i j}$ & Kronecker Delta Tensor \\
\hline$\delta$ & Boundary Layer Thickness, in \\
\hline$\delta_{0}$ & Onset Boundary Layer Thickness, in \\
\hline$\delta *$ & Boundary Layer Displacement Thickness, in \\
\hline$\varepsilon$ & Dissipation Rate of $\mathrm{k}, \mathrm{ft}^{2} / \mathrm{s}^{3}$ \\
\hline$v$ & Kinematic Viscosity, $\mathrm{ft}^{2} / \mathrm{s}$ \\
\hline$v_{t}$ & Turbulent "Eddy" Viscosity, $\mathrm{ft}^{2} / \mathrm{s}$ \\
\hline$v_{\mathrm{w}}$ & Kinematic Viscosity at $\mathrm{h}=0, \mathrm{ft}^{2} / \mathrm{s}$ \\
\hline$\theta$ & BL Momentum Thickness, in \\
\hline
\end{tabular}




\section{Experimental Arrangement}

\section{Static Test Facility}

Experimental testing was conducted in the model preparation area of the NASA Langley 16-Foot Transonic Tunnel Complex. This facility is normally used for the buildup and calibration of wind tunnel models, but can also be used for nozzle testing at static conditions. Models are mounted on a sting-strut support system in a $10 \times 29$ foot ambient test chamber, and supplied with a regulated continuous flow of clean, dry air. A control room adjacent to the test chamber offers access through a sound proof door and observation window. A complete description of this test facility is provided in reference 5 .

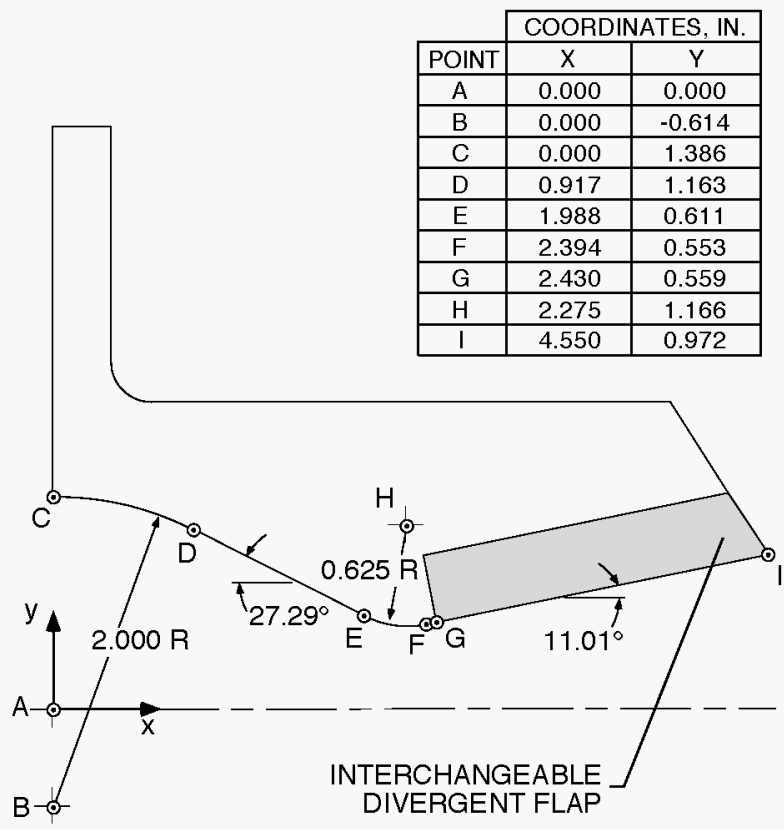

Figure 2: Nozzle Flap Geometry

\section{Test Nozzle}

The test nozzle used in this investigation was a subscale, non-axisymmetric, two-dimensional convergentdivergent (2D-CD) nozzle with a nominal throat area $\mathrm{A}_{\mathrm{t}}=4.317 \mathrm{in}^{2}$, an expansion ratio $\mathrm{A}_{\mathrm{e}} / \mathrm{A}_{\mathrm{t}}=1.797$, and $\mathrm{a}$ constant width of 3.990 inches. Based on 1D Theory, the nozzle has a design NPR of 8.78, an exit Mach number of 2.07, and a design throat Reynolds number of $3.2 \times 10^{6}$ for $p_{a}=14.85 \mathrm{psi}$. The nozzle was equipped with interchangeable divergent flaps in order to function as a testbed for various shock - boundary layer interaction control concepts, and had full length opticalquality glass sidewalls to allow for internal flow visualization and flow diagnostics. Geometric details of the nozzle are shown in figure 2, and a photo of the nozzle is given in figure 3 .

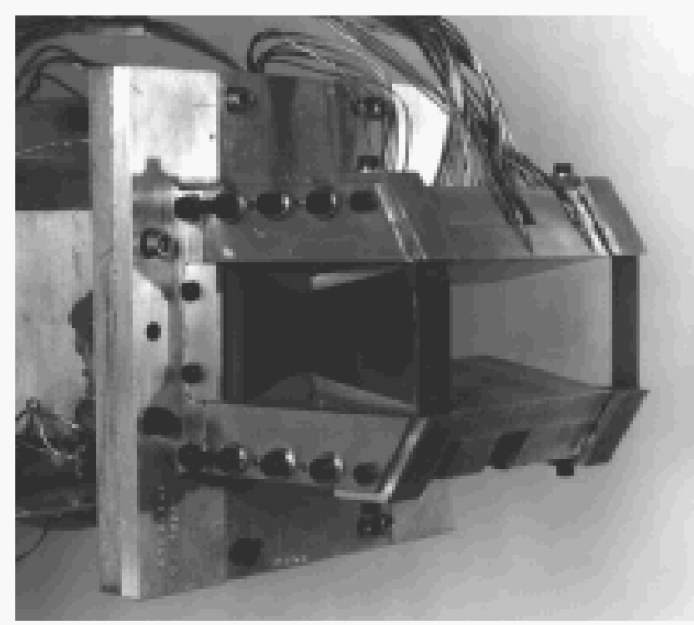

Figure 3: Test Nozzle

\section{Propulsion Simulation System}

High pressure air was supplied to the test nozzle at a stagnation temperature of about $530^{\circ} \mathrm{R}$, with flow rates up to $15 \mathrm{lbm} / \mathrm{sec}$. As shown in figure 4 , the propulsion simulation system uses choke points, plenums, turns, and flexible metal bellows to deliver air to a nozzle such that effects of momentum transfer and pressurization are minimized. This ensures that forces and moments produced by the nozzle can be accurately measured. Downstream of the flow transfer system, air passes through a circular-to-rectangular transition section, a choke plate, an instrumentation duct, and the test nozzle before exhausting to ambient back pressure.

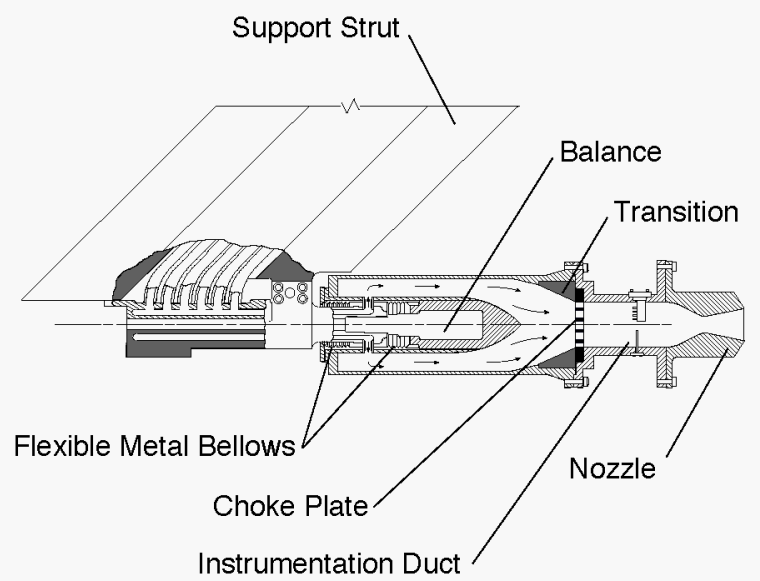

Figure 4: Propulsion Simulation System 


\section{Instrumentation}

Air flow rates were calculated from pressure and temperature measurements in a multiple critical venturi located upstream of the propulsion simulation system. Forces and moments were measured by a sixcomponent strain-gauge balance located on the propulsion simulation system centerline (see figure 3). Stagnation conditions were measured in the instrumentation duct using pitot tube rakes and pressure transducers for total pressure, and iron-constantan thermocouples for total temperature. Surface static pressures were measured on the upper nozzle flap at the centerline (convergent and divergent flap) and at 0.40 inch from the nozzle sidewall (divergent flap only). Pressure orifices were 0.020 inch in diameter and were connected to a combination of pressure transducers and an electronically scanning pressure module.

\section{Data Acquisition and Reduction}

Readings from the venturi, force balance, and pressure and temperature instrumentation were recorded simultaneously. Steady state data were acquired by averaging $\mathbf{5 0}$ frames of instantaneous data sampled at a $10 \mathrm{~Hz}$ rate. Calibration constants were applied to the raw data to obtain corrected forces, moments, pressures, and temperatures. A detailed description of the data reduction procedures used is given in reference 6 .

The following conventions were used for data reduction: Nozzle Pressure Ratio (NPR) is the ratio of jet total pressure $p_{o j}$ to atmospheric back pressure $p_{a}$, and was used to set test points. Thrust Efficiency Ratio $\left(\mathrm{F} / \mathrm{F}_{\mathrm{i}}\right)$ is the ratio of measured axial thrust $\mathrm{F}$ to ideal isentropic thrust $F_{i}$ (calculated from the measured weight flow and stagnation conditions using 1D Theory). Normalized Static Pressure $\left(\mathrm{p} / \mathrm{p}_{\mathrm{oj}}\right)$ is the measured local static pressure $p$ normalized by the jet total pressure $\mathrm{p}_{\mathrm{oj}}$.

\section{Test Schedule}

Nozzle testing was performed by taking twenty points of data over an NPR range from 1.25 to 9.5 . These limits were established by the lower and upper flow rates of the air supply system. Fifteen of the twenty data points were taken below NPR $=6$ to obtain detailed information on the overexpanded, shock-separated regime of the nozzle.

\section{Uncertainty Analysis}

An uncertainty analysis was performed by propagating measurement bias uncertainties through the data reduction equations. This analysis assumes that bias errors dominate precision errors and is based on methods presented in references 7 and 8 . For the range of test conditions encountered in this study, the uncertainty of NPR and $p / p_{\text {oj }}$ is approximately $\pm 0.28 \%$ of measured value, and the uncertainty of $\mathrm{F} / \mathrm{F}_{\mathrm{i}}$ is approximately $\pm 0.4 \%$.

\section{Flow Visualization}

A focusing schlieren system was used to visualize internal nozzle flows in this study. Based on criterion developed by Weinstein ${ }^{9}$, the system had a $133 \mathrm{~mm}$ diameter field of view, a sensitivity of 17 arcsec, a resolution of $0.25 \mathrm{~mm}$, a depth of sharp focus of 4.6 $\mathrm{mm}$, and a depth of unsharp focus of $36 \mathrm{~mm}$. The light source for the schlieren system was a xenon strobe flash tube, driven at a $30 \mathrm{~Hz}$ rate with pulses of $0.6 \mu \mathrm{sec}$ duration and 0.05 watt.sec power. The system was focused on the test nozzle centerline plane and configured for sensitivity to streamwise density gradients. A $720 \times 480$ pixel resolution video camera and $70 \mathrm{~mm}$ Hasselblad still camera recorded results.

\section{Theoretical Modeling}

Theoretical predictions were made using the NPAC code developed at NASA Langley ${ }^{10}$. This code uses an approximate theoretical method to calculate internal flow, thrust performance, and boundary layer characteristics of $2 \mathrm{D}-\mathrm{CD}$ nozzles. Internal flow and thermodynamic performance effects due to over- and underexpansion are modeled with one-dimensional compressible flow theory, and an approximate integral momentum method (based on the classic KármánPolhausen solution) is used to calculate turbulent boundary layer development and skin friction losses. An iterative algorithm couples the boundary layer solution to the 1D flow model to correct for the effects of boundary layer displacement on internal flow. Additional calculations account for exit flow angularity losses. The NPAC method has been well validated, and is capable of predicting peak thrust efficiency to within $0.1 \%$ for a wide variety of $2 \mathrm{D}-\mathrm{CD}$ nozzle shapes. For low expansion ratio nozzles, NPAC can generally predict off-design thrust efficiency to within $0.5 \%$.

\section{Computational Fluid Dynamics Simulation}

The NASA Langley Reynolds-averaged Navier Stokes (RANS) computational fluid dynamics (CFD) code PAB3D was used in conjunction with two-equation $k-\varepsilon$ turbulence closure and nonlinear algebraic Reynolds stress models to simulate separated nozzle flows in this investigation. This code has been well tested and documented for the simulation of aeropropulsive and aerodynamic flows involving separation, mixing, and other complicated phenomena ${ }^{11,12,13}$. Currently, PAB3D is ported to a number of platforms and offers a 
combination of good performance and low memory requirements. In addition to its advanced preprocessor which can handle complex geometries through multiblock general patching. PAB3D has a runtime module capable of calculating aerodynamic performance on the fly and a postprocessor used for follow-on data analysis.

\section{Flow Solver and Governing Equations}

PAB3D solves the simplified Reynolds-averaged Navier-Stokes equations in conservative form, obtained by neglecting streamwise derivatives of the viscous terms. Viscous models include coupled and uncoupled simplified Navier-Stokes and thin layer Navier-Stokes options. Roe's upwind scheme is used to evaluate the explicit part of the governing equations, and van Leer's scheme is used for the implicit part ${ }^{14}$. Diffusion terms are centrally differenced, inviscid terms are upwind differenced, and two finite volume flux-splitting schemes are used to construct the convective flux terms ${ }^{14}$. PAB3D is third-order accurate in space and first-order accurate in time. For numerical stability, various solution limiters can be used, including minmod, van Albeda, and Spekreijse-Venkat ${ }^{14}$. The code can utilize either a 2 -factor or 3-factor numerical scheme to solve the governing equations.

For the present study, the $2 \mathrm{D}$ problem was defined by $\mathrm{j}$ and $\mathrm{k}$ indices in a single $\mathrm{i}=$ constant plane (the $\mathrm{j}$ index was oriented in the streamwise flow direction). With this arrangement, explicit sweeps in the $i$ direction were not needed, and it was possible to solve the entire problem implicitly with each iteration (using the van Leer scheme). This strategy speeds convergence and reduces computational time. Based on previous experience with shock - boundary layer interaction problems, the uncoupled j-k simplified Navier-Stokes viscous option and the Spekreijse-Venkat limiter were selected.

\section{Turbulence Closure}

In simulating turbulence, transport equations for the turbulent kinetic energy per unit mass $(\mathrm{k})$ and the dissipation rate of $k(\varepsilon)$ are uncoupled from the mean flow RANS equations and can be solved with a different time step to speed convergence. These turbulence transport equations are of the standard linear form shown below, and are solved by the same numerical schemes discussed above. The constants in the $\varepsilon$ equation assume their standard values of $\mathrm{C}_{\varepsilon 1}=1.44$, $\mathrm{C}_{\varepsilon 2}=1.92, \sigma_{\mathrm{k}}=1$, and $\sigma_{\varepsilon}=1.3$.

$$
\frac{D k}{D t}=\frac{\partial}{\partial x_{j}}\left[\left(v+\frac{v_{t}}{\sigma_{k}}\right) \frac{\partial k}{\partial x_{j}}\right]-\left\langle u_{i}^{\prime} u_{j}^{\prime}\right\rangle \frac{\partial U_{i}}{\partial x_{j}}-\varepsilon
$$

$$
\begin{gathered}
\frac{D \varepsilon}{D t}=\frac{\partial}{\partial x_{j}}\left[\left(v+\frac{v_{t}}{\sigma_{\varepsilon}}\right) \frac{\partial \varepsilon}{\partial x_{j}}\right]-C_{\varepsilon 1} \frac{\varepsilon}{k}\left\langle u_{i}^{\prime} u_{j}^{\prime}\right\rangle \frac{\partial U_{i}}{\partial x_{j}}-C_{\varepsilon 2} \frac{\varepsilon^{2}}{k} \\
v_{t}=C_{\mu} \frac{k^{2}}{\varepsilon}
\end{gathered}
$$

With eddy viscosity or linear stress schemes, $\mathrm{C}_{\mu}$ is taken to be constant (usually equal to 0.09); however, this can produce undesirable and unphysical results under certain conditions. Models with constant $\mathrm{C}_{\mu}$ can predict negative values of $\mathrm{k}$ in flows with rapid strain, and can also produce non-realizable Reynolds stresses in flows with high shear, violating the Schwarz Inequality. In order to circumvent these problems, PAB3D contains several different nonlinear algebraic Reynolds stress models which were used in this study. These models give inherently better results than eddyviscosity or linear stress schemes due to the explicit modeling of effects such as relaxation, and the specific inclusion of nonlinear anisotropic effects from the mean flow strain rate and vorticity. With a nonlinear model, the calculation of six independent, realizable Reynolds stress terms is possible. This type of detail is important for simulating complicated multidimensional flows.

As an example, the algebraic Reynolds stress model of Shih, Zhu, and Lumley ${ }^{15}$ is shown below, along with it's associated equation for variable $C_{\mu}$. Note the higher order nonlinear terms involving the deviatoric mean flow strain rate $\left(\mathrm{S}^{*}\right)$ and vorticity $\left(\Omega^{*}\right)$ tensors in the stress equation. In the $C_{\mu}$ equation, $A_{0}=6.5$, and $C_{2}$, $A_{\mathrm{s}}^{*}$, and $\mathrm{U}^{*}$ involve tensor products of $\mathrm{S}^{*}$ and $\Omega^{*}$ and their variants. Further details on the variables and coefficients in this model are available in reference 15.

$$
\begin{gathered}
\left\langle u_{\mathrm{i}}^{\prime} \mathrm{u}_{\mathrm{j}}^{\prime}\right\rangle=\frac{2}{3} \mathrm{k} \delta_{\mathrm{ij}}-2 \mathrm{C}_{\mu} \frac{\mathrm{k}^{2}}{\varepsilon} \mathrm{S}_{\mathrm{ij}}^{*}+2 \mathrm{C}_{2} \frac{\mathrm{k}^{3}}{\varepsilon^{2}}\left(\Omega_{\mathrm{ik}}^{*} S_{\mathrm{kj}}^{*}-\mathrm{S}_{\mathrm{ik}}^{*} \Omega_{\mathrm{kj}}^{*}\right) \\
\mathrm{C}_{\mu}=\left(\mathrm{A}_{\mathrm{o}}+\mathrm{A}_{\mathrm{s}}^{*} \mathrm{U}^{*} \frac{\mathrm{k}}{\varepsilon}\right)^{-1}
\end{gathered}
$$

\section{Computational Domain}

Details of the full 2D computational domain are presented in figure 5 , where the multiblock grid is shown at $1 / 4$ resolution. Relative to the nozzle exit, the ambient region surrounding the nozzle extended approximately 30 throat heights downstream, 25 throat heights upstream, and 25 throat heights normal to the jet axis. 


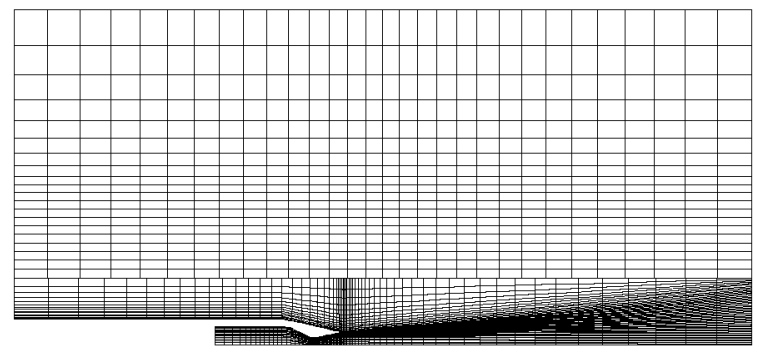

Figure 5: Computational Grid at 1/4 Resolution

The internal nozzle grid is shown at full resolution in figure 6 , and is characterized by an adaptive boundary layer grid with an expansion ratio of about $18 \%$ and a first cell height of approximately $\mathrm{y}^{+}=0.5$ (adaptive design based on boundary layer characteristics predicted by NPAC). There were approximately 40 cells in the boundary layer grid. In an attempt to capture the complicated physics of the shock boundary layer interaction process, the divergent section of the nozzle was densely gridded with cells having an aspect ratio near 1:1. An inflow duct (sized like the instrumentation duct used in the experimental study) was located upstream of the nozzle.

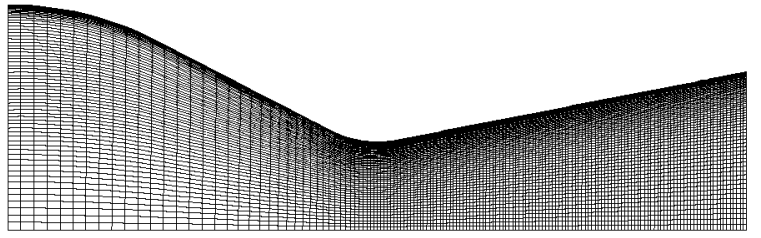

Figure 6: Nozzle Grid at Full Resolution

\section{Initial and Boundary Conditions}

As shown in figure 7 , stagnation conditions were applied to the left face of the inflow duct upstream of the nozzle, and were chosen to match experimental conditions for $\mathrm{T}_{\mathrm{oj}}$ and $\mathrm{p}_{\mathrm{oj}}$. In addition, an initial Mach number was specified in the inflow block and nozzle to start the solution. The static ambient region surrounding the nozzle was defined by a subsonic inflow condition $\left(T_{a}=530^{\circ} \mathrm{R}, \mathrm{p}_{\mathrm{a}}=14.85 \mathrm{psi}, \mathrm{M}_{\mathrm{a}}=0.025\right)$ on the left face, a characteristic boundary condition on the top face, and a smart boundary condition on the right face that switched between constant pressure outflow (subsonic) and first order extrapolation (supersonic) depending on the local Mach number. All solid walls were treated as no-slip adiabatic surfaces, and the bottom of the entire domain was defined by a slip wall (acting as a symmetry boundary condition).

In order to initialize the turbulence transport equations and ensure the formation of a turbulent boundary layer in the test nozzle, a wall "trip" point was located near the beginning of the inflow duct. At this point, $\mathrm{k}$ was specified based on calculations involving the mean flow velocity and vorticity and a user specified intensity ratio. A corresponding value of $\varepsilon$ was calculated based on the simplifying and reasonable assumption that the production of TKE was equal to the dissipation of TKE at the trip point.

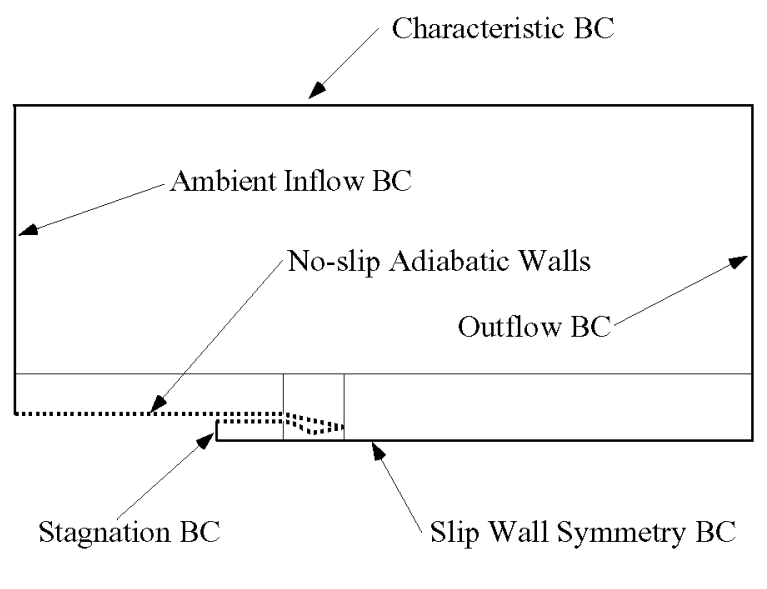

Figure 7: Boundary Conditions

\section{Solution Procedure and Postprocessing}

All solutions presented in this paper were obtained by running PAB3D on an SGI Octane workstation with a 195Mhz MIPS-R10000 CPU and $896 \mathrm{MB}$ of RAM. To speed convergence and evaluate possible grid dependence, mesh sequencing was used to evolve solutions through coarse ( $1 / 4$ resolution), medium ( $1 / 2$ resolution), and fine (full resolution) grids. Local timestepping was used with global CFL numbers ranging from 1 to 5 . Depending on the NPR and complexity of flow in the nozzle, it took 5000-14000 iterations and 8-26 hours of CPU time to obtain a fully converged 2D solution. Convergence was judged by tracking an integrated $\mathrm{F} / \mathrm{F}_{\mathrm{i}}$ calculation until it settled to three decimal places over at least 1000 iterations.

The inline performance module of PAB3D was used in conjunction with an independent postprocessor to compute thrust efficiency by integrating pressure and momentum over a fixed control volume. The postprocessor was also used to extract internal nozzle surface pressure data and construct schlieren-like images of the nozzle flow. These images were obtained by calculating the density gradient and combining it with a simulated optical "cutoff" effect. All images presented in this paper were generated with a root mean square average of horizontal and vertical cutoffs, and thus show sensitivity to both streamwise and transverse density gradients. 


\section{Experimental Results}

In this section, experimental results will be presented in terms of internal flow features (static pressure measurements and schlieren flow visualization) and thrust performance. Because the test nozzle had glass sidewalls that flexed slightly under pressurization, it was not possible to accurately measure discharge coefficient and these data are not presented.

\section{Internal Flow}

Normalized experimental centerline static pressures $\left(\mathrm{p} / \mathrm{p}_{\mathrm{oj}}\right)$ are presented in figure 8 , plotted against nondimensional streamwise location. Results are representative of classic CD nozzle flow ${ }^{16}$. For the first data points at NPRs 1.25 and 1.4, pressure data indicate choked, internally overexpanded flow with a weak shock near the geometric throat. Flow downstream of the shock appears to recover to ambient pressure $\left(\mathrm{p} / \mathrm{p}_{\mathrm{oj}}=1 / \mathrm{NPR}\right)$ in a smooth continuous fashion. Focusing schlieren flow visualization obtained at $\mathrm{NPR}=1.4$ is presented in figure 9, and shows a weak, almost normal shock downstream of the nozzle throat with little or no lambda foot structure evident. This shock - boundary layer interaction is characteristic of a weak shock $\left(\mathrm{M}_{1} \approx 1.2\right.$, estimated from $\left.\mathrm{p} / \mathrm{p}_{\mathrm{oj}}\right)$ and a thin boundary layer.

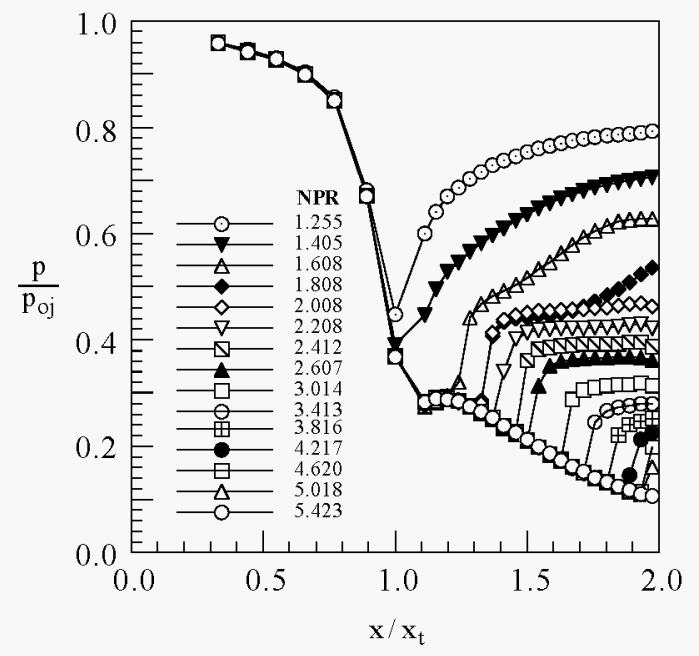

Figure 8: Experimental Centerline Pressure Data

As seen in figure 8 , the discontinuous nature of the pressure distribution at NPR $=1.6$ indicates that the strength of the nozzle shock increased $\left(M_{1} \approx 1.4\right)$, and the inflection point in the recovery curve at $\mathrm{x} / \mathrm{x}_{\mathrm{t}}=1.3$ indicates that separation occurred, though it was not severe. By NPR $=1.8$, the upstream shock Mach number was approximately 1.5 , and shock induced separation began to significantly affect nozzle flow. At this NPR, there are strong signs of a separation bubble, with minimal pressure recovery from the shock location at $\mathrm{x} / \mathrm{x}_{\mathrm{t}}=1.3$ out to $\mathrm{x} / \mathrm{x}_{\mathrm{t}}=1.6$, and strong recovery occurring over the remaining length of the nozzle. Figure 10 shows the nozzle shock at NPR $=1.8$ with a small lambda foot, and also shows signs of unstable nozzle flow; the schlieren photograph imaged the shock in two positions over a $0.6 \mu \mathrm{sec}$ duration, indicating that the separation was unsteady or transitory.



Figure 9: Schlieren Flow Visualization at $N P R=1.4$

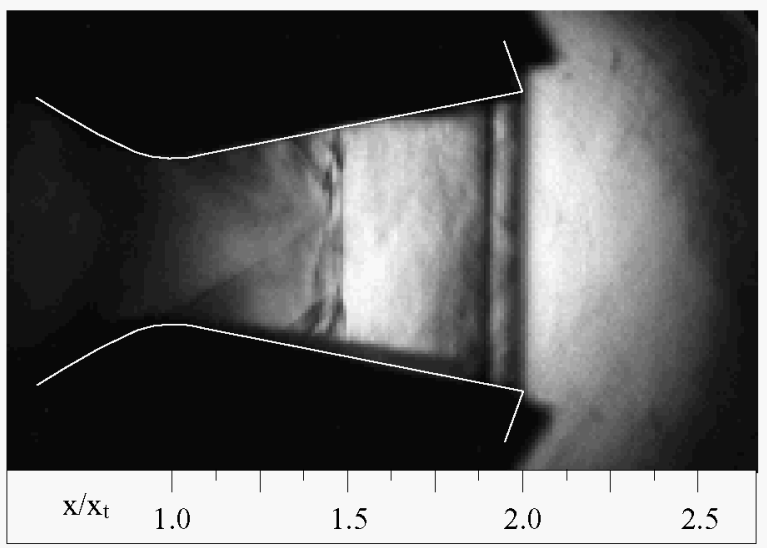

Figure 10: Schlieren Flow Visualization at $N P R=1.8$

As shown by pressure data in figure 8 , an increase in NPR to 2.0 did not change shock location or strength, but flow fully detached and there was almost no pressure recovery downstream of the shock. This result is of critical importance, because it serves to illustrate the relationship between NPR, shock - boundary layer interaction, and separation. For this NPR, nozzle flow adjusted to exit conditions by completely detaching past the shock; internal conditions up to and through the shock were nearly identical to those of the previous NPR. Thus, separation that occurred at NPR $=2.0$ was probably not the result of a stronger shock - boundary layer interaction, but instead came about through the natural tendency of an overexpanded nozzle flow to separate, thereby "adjusting" to a lower expansion ratio 
geometry. Among other things, this suggests that the shock - boundary layer interaction is not so much the "cause" of separation, but is more of a mutually dependent "result".

Schlieren flow visualization at $\mathrm{NPR}=2.0$ in figure 11 shows the nozzle shock with a pronounced lambda foot system and fully detached separation extending from the leading lambda shock downstream past the nozzle exit. That the appearance of a large lambda foot coincided with the onset of fully detached separation inside the nozzle is an important observation, for it shows that the size and extent of a lambda foot is more a result of separation effects than it is of basic shock boundary layer interaction conditions such as onset Mach number or boundary layer state. This makes sense, since a fully detached region would impose stronger turning requirements on nozzle flow than a closed separation bubble and would require a bigger oblique shock system. As the separation point became the effective nozzle exit, the lambda shock system adjusted to satisfy continuity of pressure and flow direction in the new effective geometry.

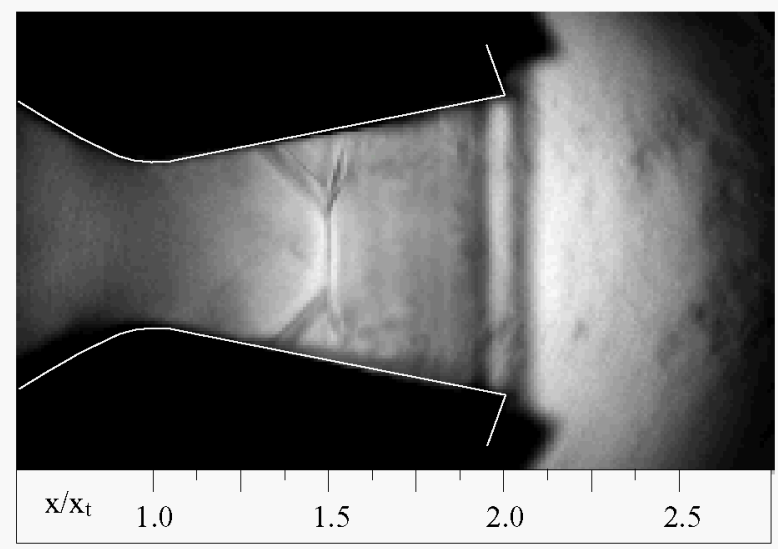

Figure 11: Schlieren Flow Visualization at $N P R=2.0$

Fully detached separation occurred for subsequent NPRs past 2.0. Figure 12 shows the shock at NPR=2.4 with a well defined lambda foot and separation, and figure 13 shows the same at NPR=3.0. By NPR=3.4, the lambda shock foot had grown significantly, such that the main shock and trailing lambda foot were outside the "physical" nozzle, as shown in figure 14 . At this NPR, flow past the separation point showed strong resemblance to externally overexpanded flow; the jet plume necked down between the leading and trailing lambda foot, and there was an expansion fan emanating from each trailing lambda foot as it intersected the free shear layer. This indicates that not only was the separation point behaving like the nozzle exit, but flow past this point was overexpanding externally, which would occur in a lower expansion ratio nozzle at the same NPR.

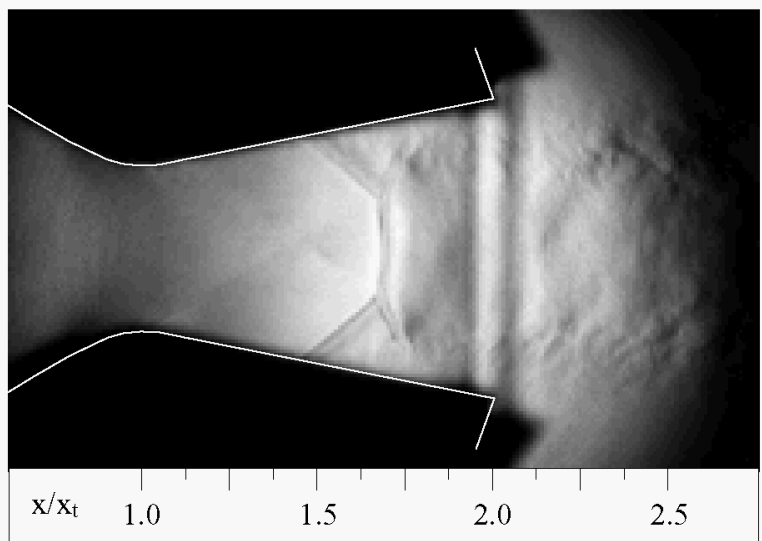

Figure 12: Schlieren Flow Visualization at NPR $=2.4$

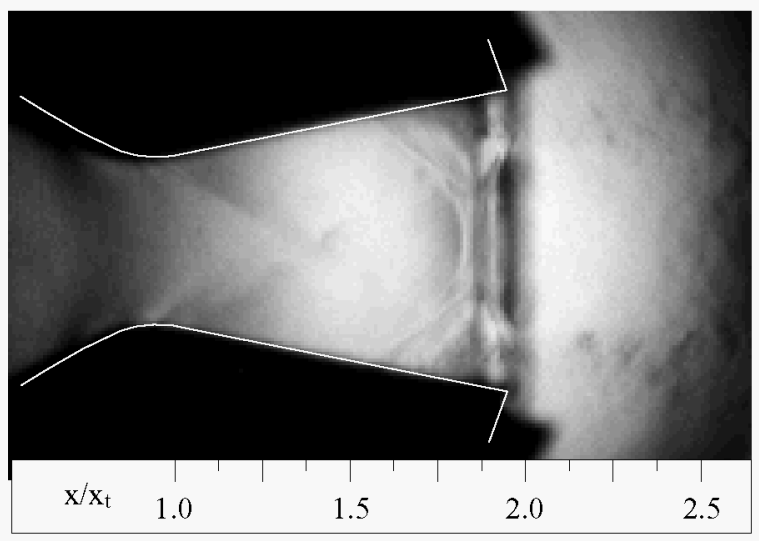

Figure 13: Schlieren Flow Visualization at $N P R=3.0$

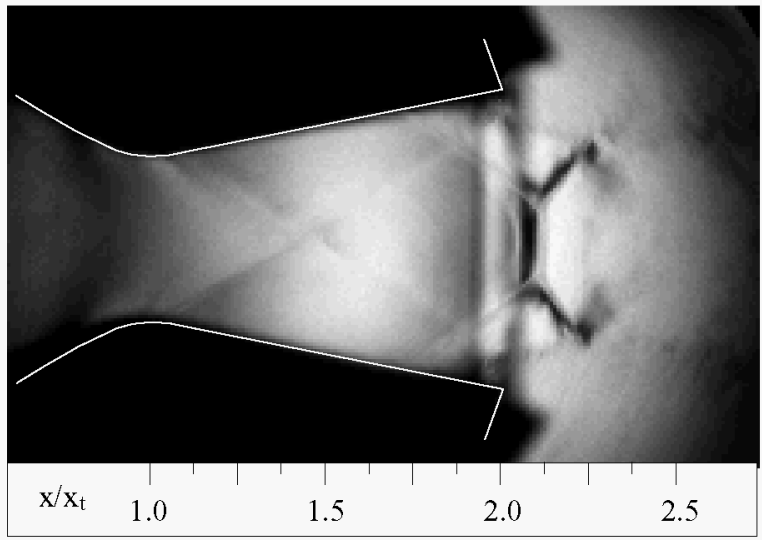

Figure 14: Schlieren Flow Visualization at NPR=3.4

The leading lambda foot worked its way out of the nozzle with increasing NPR, and pressure data in figure 8 show the nozzle to be shock free by NPR=5.4. As shown in figure 15, pressures fell on the same curve above NPR $=5.4$, indicating that internal flow was independent of NPR in this range. By comparing experimental data with a theoretical pressure distribution, one feature clearly evident in figure 15 is 
the "vena contracta" effect, which occurs when acceleration causes flow to overshoot the nozzle throat radius. This causes flow to reach sonic velocity upstream of the geometric throat, and leads to local overexpansion and compression, evidenced by the dip and bump in pressure data from $\mathrm{x} / \mathrm{x}_{\mathrm{t}} \approx 0.8$ to $\mathrm{x} / \mathrm{x}_{\mathrm{t}} \approx 1.3$. High-sensitivity schlieren flow visualization at $\mathrm{NPR}=8.95$ (near design) is presented in figure 16 , and shows the skewed, bell-shaped sonic line (dark band) upstream of the geometric nozzle throat, the local overexpansion (dark regions) near the throat, and oblique shocks marking the recompression. These features were present for all NPRs above 1.6.

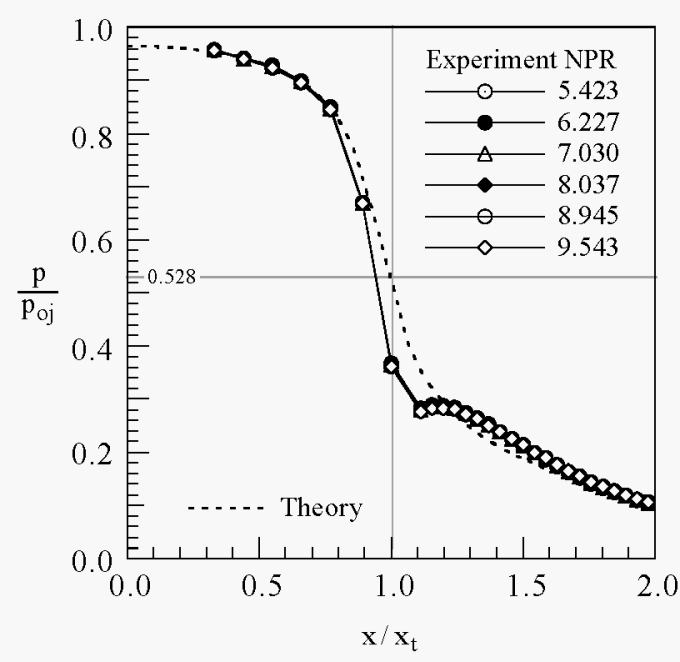

Figure 15: Centerline Pressures for NPR $\geq 5.4$



Figure 16: Schlieren Flow Visualization at NPR $=8.95$

\section{Experimental Sideline Pressures}

Experimental divergent flap sideline pressures are compared to centerline pressures at selected NPRs in figure 17. At NPR $=1.8$, there are noticeable differences between sideline and centerline pressure in terms of shock location and downstream pressure recovery, indicating that nozzle flow was highly threedimensional (3D) and the shock was non-planar. For $\mathrm{NPR}=2.4$ and above, sideline and centerline exit pressures were equal, and by NPR $=3.0$, sideline and centerline shock locations became nearly equal and remained that way for all higher NPRs. Sideline and centerline pressure distributions showed good agreement at NPR $=3.0$, indicating that flow was well behaved and nearly two-dimensional. At NPR=5.4 (shock free nozzle), sideline and centerline pressures fall on the same curve.

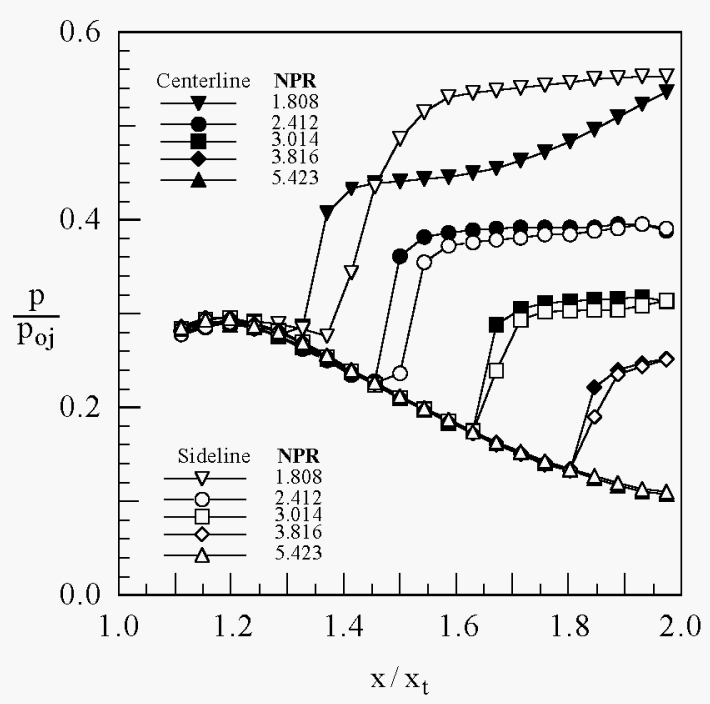

Figure 17: Centerline - Sideline Pressure Comparison

\section{Performance}

Thrust efficiency results are shown in figure 18, where the measured thrust ratio $F / F_{i}$ is plotted versus NPR and accompanied by a theoretical curve (predicted by NPAC) for comparison. Near the design pressure ratio of 8.78, measured thrust efficiency reached a maximum of $\mathrm{F} / \mathrm{F}_{\mathrm{i}}=0.986$, which is consistent with the $0.985-0.990$ range reported in other studies ${ }^{17,18}$, and in excellent agreement with the theoretical prediction of 0.986 . This indicates that on-design losses were likely due to the same effects modeled by the NPAC code at this fully expanded condition; namely, skin friction, boundary layer displacement, and flow angularity. 


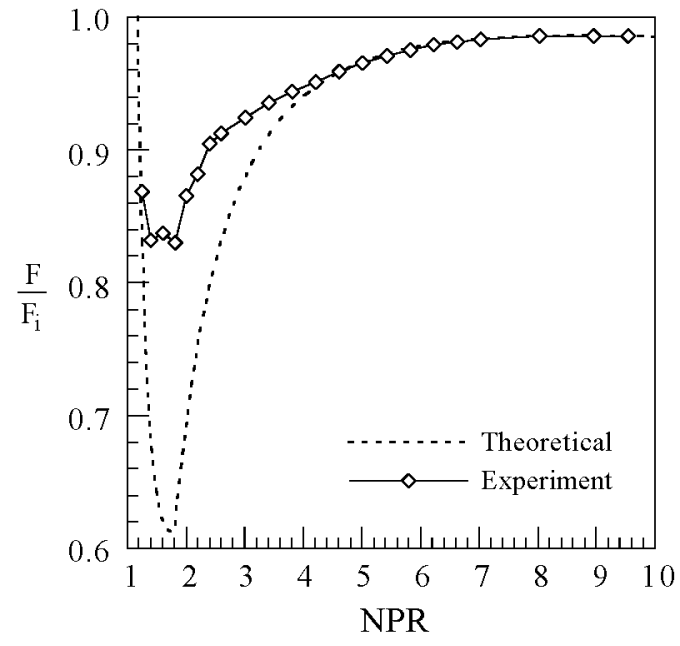

Figure 18: Experimental and Theoretical Thrust Efficiency Comparison

Below the design point of the nozzle, measured thrust efficiency decreased, closely following the theoretical prediction down to about $\mathrm{NPR}=4.6$. Again using the NPAC model for guidance, this indicates that internal performance effects and losses were constant in this range, and that thrust efficiency was governed by the thermodynamics of flow in an overexpanded CD nozzle. The fact that this agreement extended down to $\mathrm{NPR}=4.6$ correlates well with pressure data and earlier discussion that indicated the nozzle was approaching a shock free condition near this point.

Below NPR $=4.6$, measured thrust efficiency rose above the theoretical prediction and remained there for all lower NPRs before returning to the theoretical curve at $\mathrm{NPR}=1.25$. At it's best point, measured thrust efficiency showed a $22 \%$ increase over theoretical thrust efficiency at $\mathrm{NPR}=1.6$. As supported by previous discussion, this behavior corresponds to the fact that shock induced boundary layer separation was "altering" the effective nozzle geometry to one with a more efficient, lower expansion ratio. This was most noticeable at lower NPRs where the nozzle shock was further upstream and separation had a bigger effect on nozzle flow and the effective expansion ratio.

Several other observations round out this discussion. First, just as the high end departure from the theoretical curve occurred at NPR $=4.6$ due to the "end" of separated flow, the low end departure from the theoretical curve occurred around NPRs 1.4-1.8, where early stages of separation were present. Note that measured $\mathrm{F} / \mathrm{F}_{\mathrm{i}}$ was nearly constant in this range, as the nozzle was going through stages of "transitory" separation. In addition, the marked increase in measured $\mathrm{F} / \mathrm{F}_{\mathrm{i}}$ as NPR went from 1.8 to 2.0 corresponds to the onset of fully detached separation in the nozzle, and represents the single largest NPR to NPR leap in thrust efficiency measured (as evidenced by the slope of the $\mathrm{F} / \mathrm{F}_{\mathrm{i}}$ curve) -- an increase of some $3.5 \%$. Obviously, this confirms the fact that the nozzle flow went through a dramatic change at this point.

\section{Algebraic Reynolds Stress Model Selection}

Prior to conducting a detailed computational analysis, test cases were run at NPR $=3.0$ in order to assess the performance of various algebraic Reynolds stress (ARS) models using experimental centerline pressure data as the criterion. At this NPR, experimental results showed a good shock - boundary layer interaction, and pressure data indicated that nozzle flow was nearly $2 \mathrm{D}$. Thus, NPR $=3.0$ represented a challenging test case that was consistent with the $2 \mathrm{D}$ computational model.



Figure 19: Comparison of Experimental and Computational Pressure Data at $N P R=3.0$

Three ARS models were evaluated in this test study: the Shih-Zhu-Lumley (SZL) model ${ }^{15}$, the Gatski-Speziale (GS) model $^{19}$, and the Girimaji model ${ }^{20}$. Figure 19 presents a comparison of computational and centerline experimental pressures over the entire nozzle length at $\mathrm{NPR}=3.0$. All three ARS models showed excellent agreement with experimental pressures upstream and immediately downstream of the nozzle throat. Further downstream, all three ARS models appeared to model the shock - boundary layer interaction well. Figure 20 presents a close-up comparison in that region, and shows that the SZL model did the best overall job of placing the nozzle shock and modeling the downstream separation. Thus, it was selected for further use. 


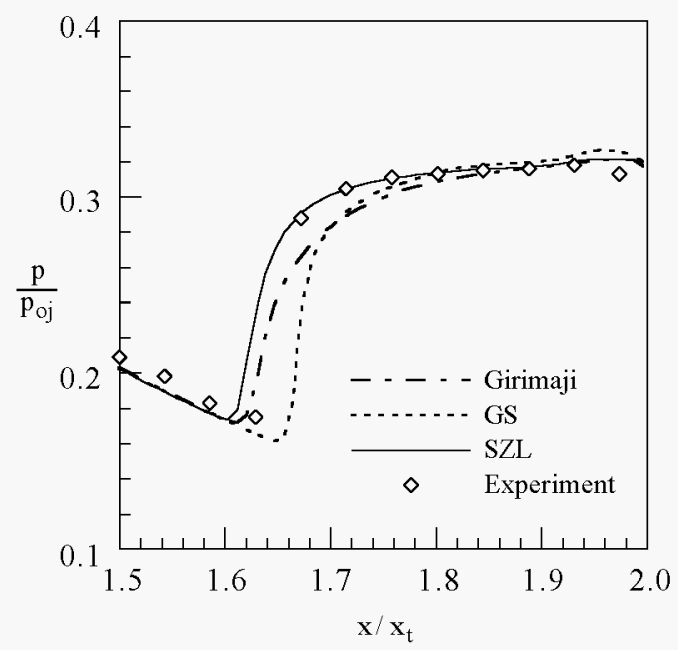

Figure 20: Comparison of Experimental and Computational Pressure Data at $N P R=3.0$

\section{Computational Results}

Computational simulations were run at the following nozzle pressure ratios: $1.25,1.4,1.6,1.8,2.0,2.2,2.4$, 3.0, 3.8, 4.6, 5.4, 7.0, and 8.78. These conditions were chosen for detailed comparison with experimental data.

\section{Internal Flow}

Low NPR pressure results from the computational simulation are compared with experimental centerline pressure data at selected NPRs in figure 21 . Note that experimental data can be keyed to it's corresponding computational curve in this figure by looking at the exit pressure $\left(\mathrm{p} / \mathrm{p}_{\mathrm{oj}}=1 / \mathrm{NPR}\right)$. At NPRs below 2.0, there is very poor agreement between experimental and computational results, with the computational simulation predicting significantly more separation in this transitory regime. A computational schlieren image at NPR $=1.4$ is presented in figure 22. Compared to its experimental counterpart in figure 9, shock structure is similar, but flow is seen to detach immediately downstream of the nozzle throat. In contrast, the experimental image shows that flow was indeed attached.

Additional CFD schlieren images in figures 23, 24, and 25 show that the computational simulation began to form a lambda shock structure as early as NPR $=1.6$, and by $\mathrm{NPR}=1.8$, the lambda system was well developed. This is in stark contrast to experimental results which indicated the presence of a transitory shock structure and transitory separation up to $\mathrm{NPR}=1.8$. In addition, the dramatic change in internal flow observed with the onset of full separation as NPR was increased from 1.8 to 2.0 in the experiment is clearly missing in the computational simulation.

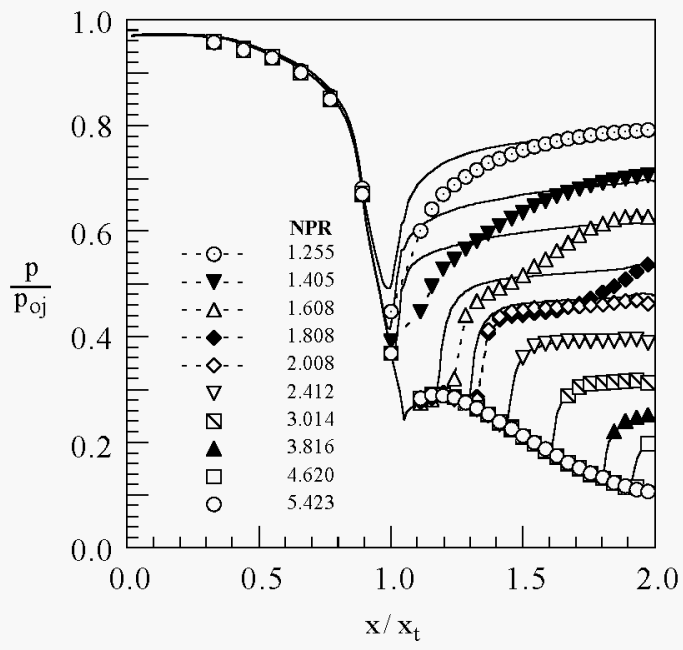

Figure 21: Low NPR Pressure Comparison

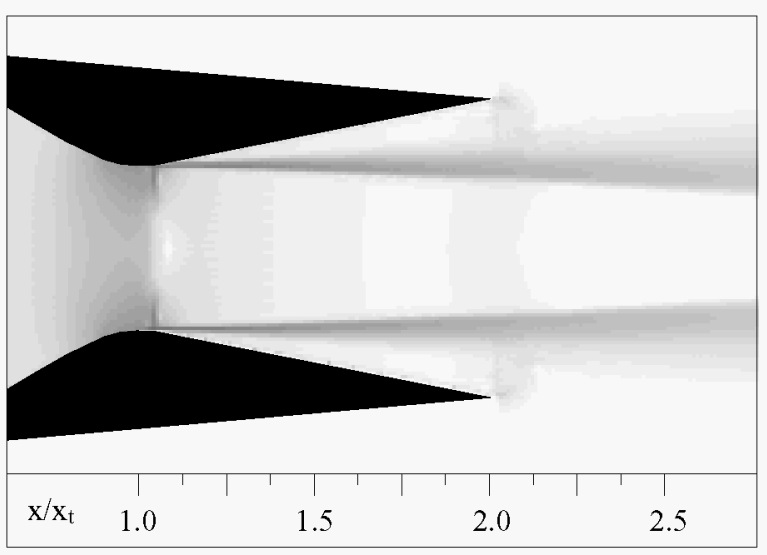

Figure 22: CFD Schlieren at $N P R=1.4$

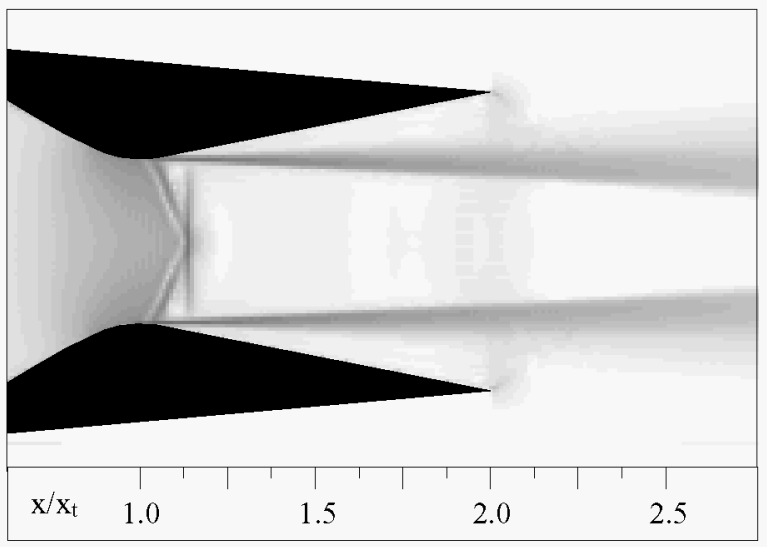

Figure 23: $C F D$ Schlieren at $N P R=1.6$ 


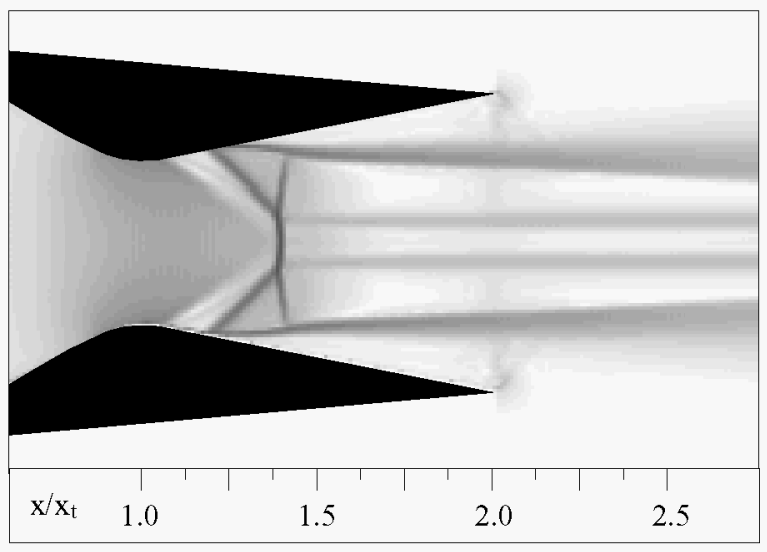

Figure 24: $C F D$ Schlieren at $N P R=1.8$

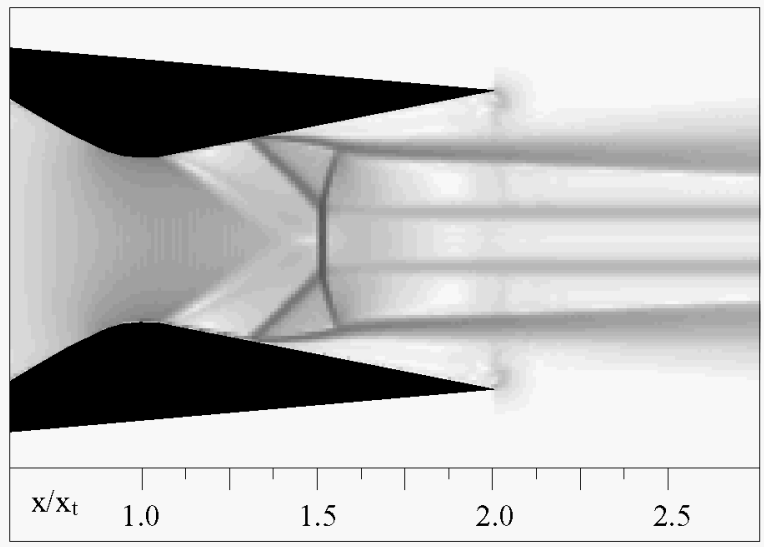

Figure 25: $C F D$ Schlieren at $N P R=2.0$

At NPR $=2.0$, experimental and computational pressure results begin to converge, and they are in very good agreement for NPRs 2.4 and up where both the experiment and computation were in a fully detached flow regime. Schlieren images at NPRs 2.0, 2.4, and 3.0 in figures 25-27 show good qualitative agreement with their experimental counterparts in figures 11-13, though the computational simulation is seen to predict more of a "stretched" shock structure at the higher NPRs (this will be studied in detail in a later section). The CFD schlieren images at NPRs 1.8, 2,0, 2.4, and 3.0 all show the ghost image of a small recirculation eddy at the nozzle exit, which suggests the presence of a double-eddy separation structure, and would explain the "wiggles" in experimental and computational surface pressure data near the exit.

The low NPR internal flow discrepancies noted between experiment and computation could be due to a number of things, but the most likely factor is the 2D nature of the computational simulation. While comparison is being made with experimental data and flow visualization from the centerline plane of the nozzle, where flow should be 2D in nature (based on symmetry), it is important to realize that "local" flow at this 2D centerline plane is the result of a "global" 3D nozzle flow. Experimental data indicated that nozzle flow was highly 3D at low NPRs (NPR $\leq 2.4$ ), which would certainly have an impact on flow at the nozzle centerline. In addition, the onset of separation is known to be a highly 3D process in many flows (though the cause of separation is usually 2D in nature), and it would seem that nozzle flows are no exception. That the flow began to take on a $2 \mathrm{D}$ nature at around $\mathrm{NPR}=2.4$, where experiment and computation begin to agree, further corroborates this argument and indicates that a 3D simulation is necessary to correctly model nozzle flow at low NPRs.

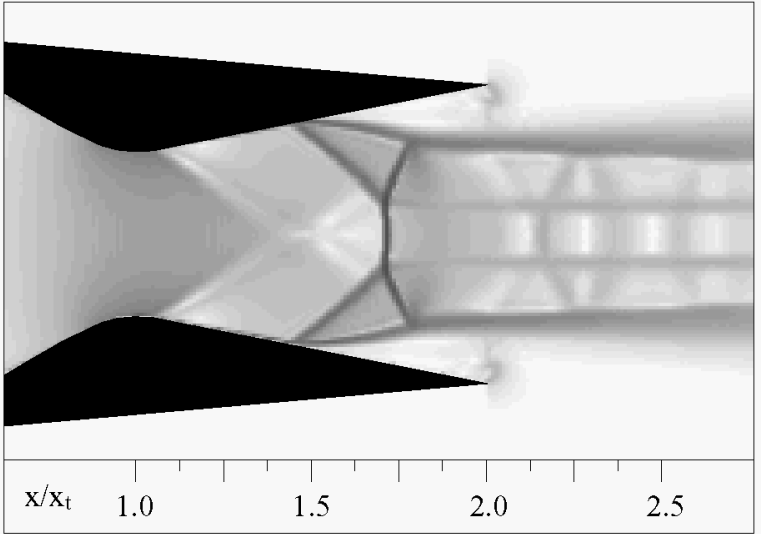

Figure 26: $C F D$ Schlieren at $N P R=2.4$

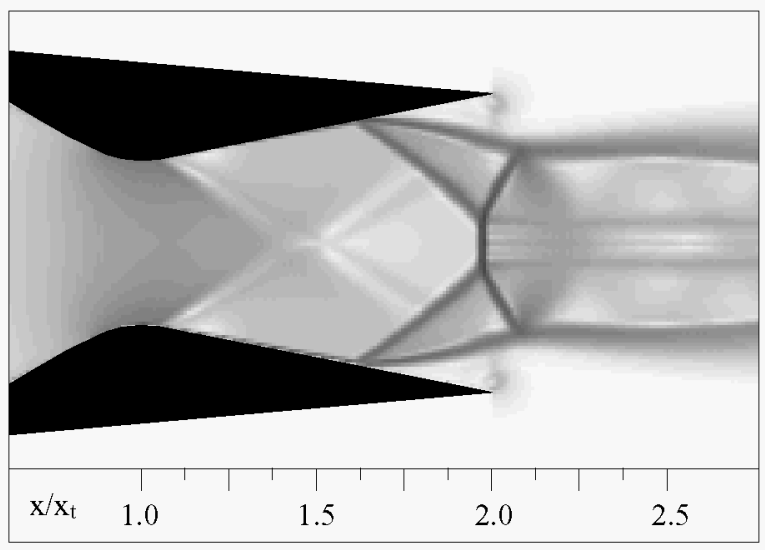

Figure 27: $C F D$ Schlieren at $N P R=3.0$

Like the experiment, computational results show that the nozzle was shock free by NPR=5.4. A comparison of high NPR pressure data with theory in figure 28 shows good agreement between the experiment and the computation (at NPRs 5.4, 7.0, 8.78), and the resolution of the computational simulation more clearly defines the extreme behavior of the vena contracta effect at the nozzle throat. Computational schlieren flow visualization at NPR $=8.78$ is presented in figure 29 , and shows excellent similarity to the corresponding experimental image in figure 16 . 


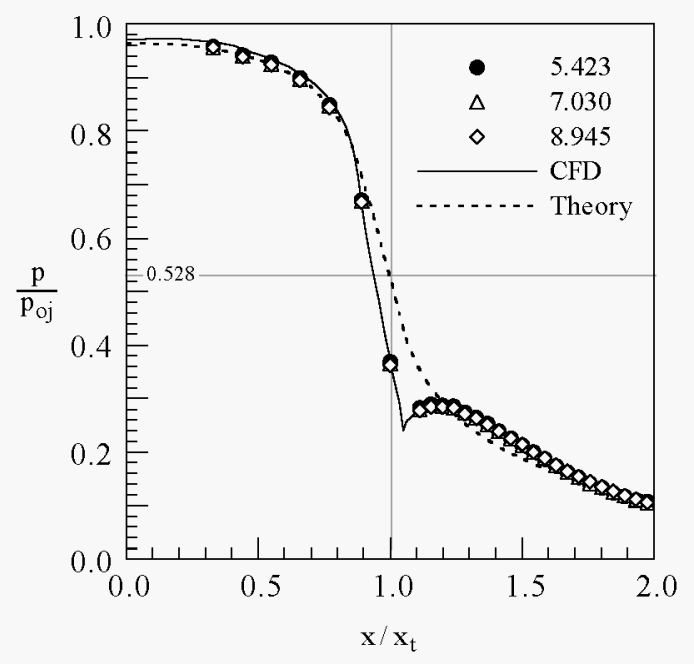

Figure 28: High NPR Pressure Comparison

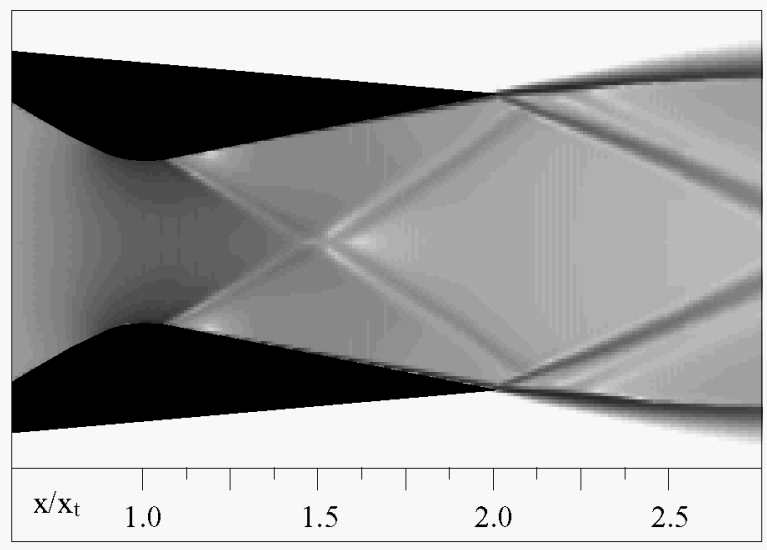

Figure 29: CFD Schlieren at $N P R=8.78$

\section{Performance}

A comparison of experimental, computational, and theoretical thrust efficiency is presented in figure 30 . As seen, the computational simulation did a very good job of modeling the overall thrust efficiency trend. It did mispredict the on-design thrust efficiency, giving a peak $F / F_{i}$ of 0.993 versus 0.986 for experiment and theory, but this is to be expected since the 2D computational model does not account for viscous effects on the nozzle sidewalls. The CFD postprocessor was used to estimate this effect by including the wetted area of the nozzle sidewalls in a skin friction calculation, and this brought the peak thrust efficiency down to 0.988 , relatively close to the experimental measurement and theoretical prediction.

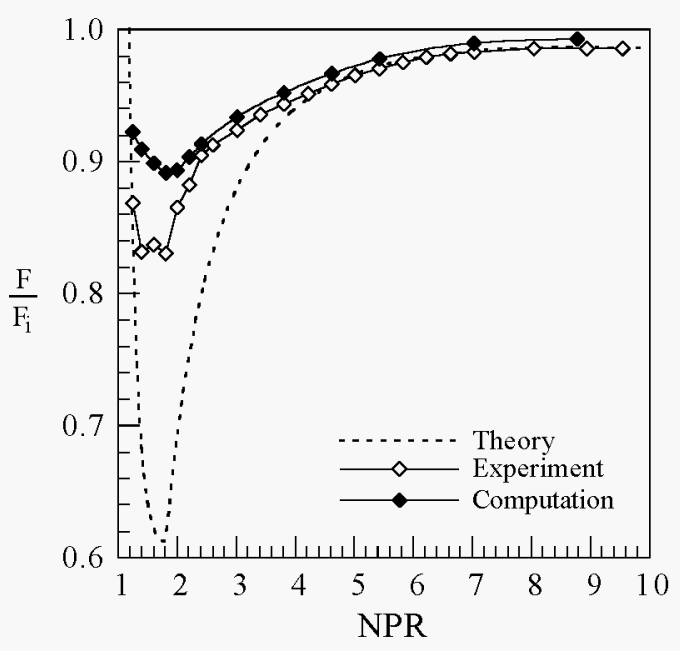

Figure 30: Thrust Efficiency Comparison

When the on-design $F / F_{i}$ shift is accounted for (graphically, this can be visualized by sliding the computational curve down to a peak of $F / F_{i}=0.986$ ), the computation still predicts up to $6 \%$ higher $\mathrm{F} / \mathrm{F}_{\mathrm{i}}$ values in the low NPR range compared with experiment. This is a direct result of the fully detached separation seen in the computational simulation but not present in the experiment. In a way this is useful, for it illustrates potential performance benefits that could be obtained in a real nozzle by encouraging fully detached separation at each off-design NPR (this is easily done using concepts discussed in reference 2). With this type of control strategy, the entire overexpanded range of nozzle performance would be within $10 \%$ of the peak thrust efficiency, which may allow a fixed geometry nozzle to cover an entire flight mission more efficiently than a mechanical system due to reductions in weight and complexity.

\section{Separation Correlation Map}

In order to map out the separated flow characteristics of a nozzle in a summary fashion, it useful to correlate the separation pressure ratio $\mathrm{p}_{\mathrm{s}} / \mathrm{p}_{\mathrm{a}}$ to the separation Mach number $M_{s}$, where $p_{s}$ and $M_{s}$ are the undisturbed pressure and Mach number upstream of the shock boundary layer interaction. A plot of this correlation is given in Figure 31 for experimental data, computational results, NPAC 1D-inviscid theory, and the empirically based theory of Reshotko and Tucker ${ }^{21}$. In the latter method, incompressible turbulent boundary layer separation criteria are corrected by a compressibility factor to predict the separation correlation. With NPAC modeling fully attached inviscid flow and the Reshotko-Tucker (R-T) method assuming fully detached separation, the two theories should bracket the range of conditions encountered in this study. 


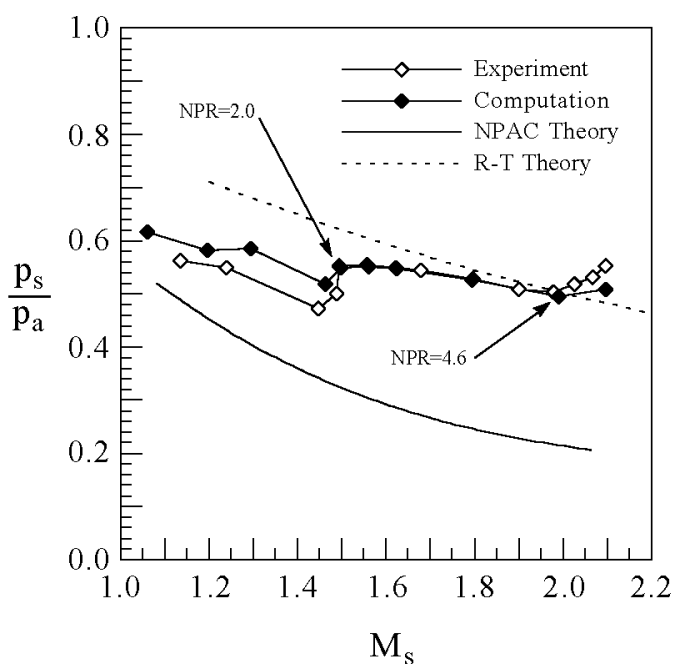

Figure 31: Separation Correlation Map

Note that the data points in Figure 31 progress from left to right in the order of ascending NPR. As expected, low NPR points for the experimental data show that the flow was in a state of partial separation which ended as the correlation moved up towards the R-T curve at $\mathrm{NPR}=2.0$. Surprisingly, computational data show similar low NPR behavior, even though CFD schlieren images indicate that flow was clearly detached in the simulation. This may indicate that there was enough pressure recovery in the separation region to lower $\mathrm{p}_{\mathrm{s}}$ back towards an attached state. At NPR $=2.0$, the computational correlation also jumps towards the fully separated limit, and then matches the experimental data out to NPR $=4.6$ as both correlations approach the R-T curve. Past that point, the experimental and computational correlations diverge from each other and the R-T curve as the nozzle tends towards a shock-free condition.

\section{Shock - Boundary Layer Interaction at NPR=2.4}

In this section, the shock - boundary layer interaction and nozzle separation at NPR $=2.4$ will be looked at in detail. Boundary layer characteristics, pressure distributions, and shock structure will be examined using a combination of experimental, computational, and theoretical results.

\section{Boundary Layer Characteristics}

Boundary layer characteristics for the nozzle flow are presented in figures $32-37$, using results from both the theoretical prediction and the computational simulation at $\mathrm{NPR}=2.4$. In each plot, the instrumentation duct starts at $x / x_{t}=-2.2$, the nozzle entrance is at $x / x_{t}=0$, and the nozzle exit is at $x / x_{t}=2$. Note that theory predicts a shock free nozzle at this NPR. To gain a better understanding of the nozzle boundary layer in the absence of a shock, an additional computational simulation was run with the same stagnation conditions $\left(p_{\mathrm{oj}}, \mathrm{T}_{\mathrm{oj}}\right)$ as the NPR=2.4 case, but with a design NPR of 8.78 (obtained by lowering the ambient back pressure). This ensured that Reynolds number was consistent with the NPR $=2.4$ case. In figures $32-36$, computational results at NPR $=2.4$ end near the shock - boundary layer interaction at $\mathrm{x} / \mathrm{x}_{\mathrm{t}} \approx 1.45$ since it was not possible to calculate boundary layer parameters past this point.

Local Reynolds number $\mathrm{Re}_{\ell}$ (based on surface arclength) is plotted versus $\mathrm{x} / \mathrm{x}_{\mathrm{t}}$ in figure 32 . As expected, the NPR $=2.4$ and shock-free computational curves are identical up to the shock - boundary layer interaction. Overall, there is good agreement between computational and theoretical results, with the exception of vena contracta induced discrepancies at the nozzle throat (from which the computational data recovers back towards the theoretical prediction). Both methods show a local Reynolds number of about $3.6 \times 10^{6}$ (or $5 \times 10^{6}$ per foot) going into the shock boundary layer interaction region.

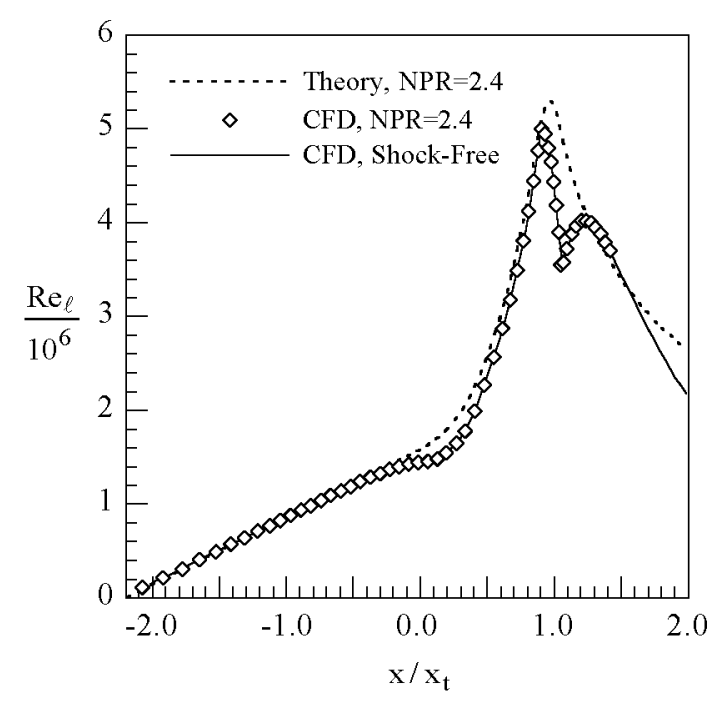

Figure 32: Local Reynolds Number

Computational turbulent kinetic energy per unit mass (k) in the boundary layer is plotted in figure 33 . Here the peak value of $\mathbf{k}$ at each streamwise location is normalized by the lower limit of $\mathrm{k}$ used in the computation. The turbulent trip point in the nozzle instrumentation duct is clearly visible at $\mathrm{x} / \mathrm{x}_{\mathrm{t}} \approx-1.6$, but it appears that the boundary layer had already transitioned to turbulence near the beginning of the duct (under certain conditions, algebraic Reynolds stress models often exhibit this type of self-transition). Downstream of the trip, the boundary layer resumed its equilibrium turbulence level in the constant area duct $(M \approx 0.23)$, and then turbulence kinetic energy grew dramatically as the boundary layer developed in the nozzle. 


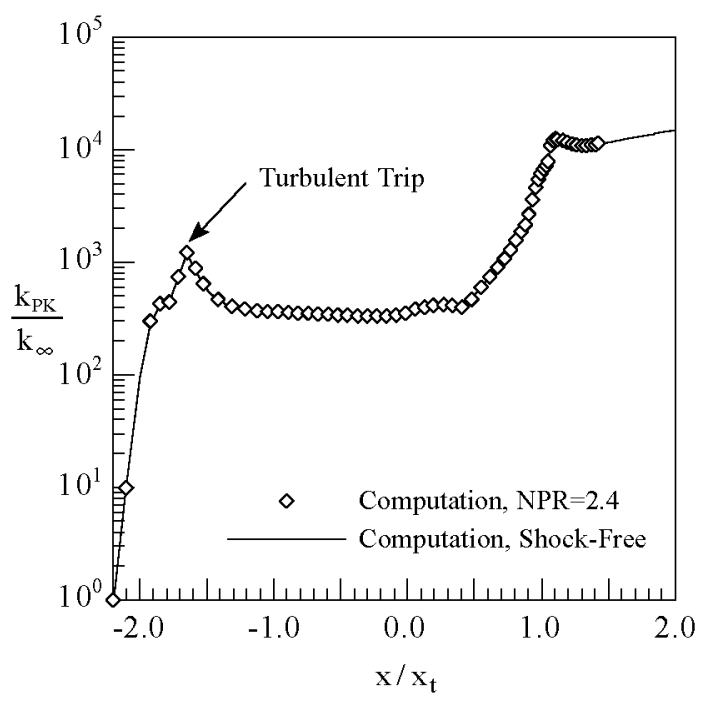

Figure 33: TKE Development

Boundary layer thickness development is presented in figure 34 , where the boundary layer edge was defined by the point at which $\mathbf{u} / \mathbf{u}_{\mathrm{e}}=0.995$. The theoretical prediction and computational simulation are in reasonably good agreement throughout the instrumentation duct and convergent section of the nozzle, and then begin to diverge downstream of the nozzle throat. Much of the difference can be attributed to the vena contracta effect present in the computational simulation, which causes the boundary layer to thin out dramatically at the nozzle throat. Going into the shock - boundary layer interaction region at $\mathrm{x} / \mathrm{x}_{\mathrm{t}} \approx 1.45$, the computational simulation predicts an onset boundary layer thickness of $\delta_{0} \approx 0.018$ in. (about $2.6 \%$ of the total nozzle height).

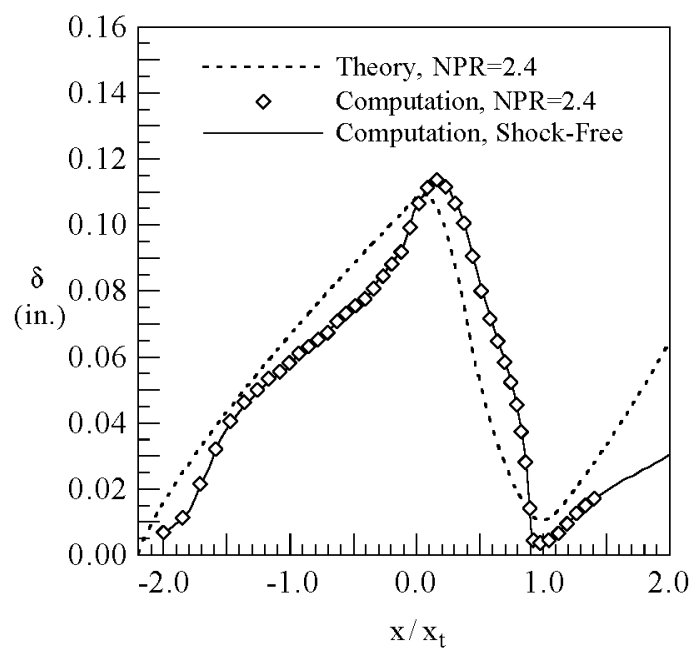

Figure 34: Boundary Layer Development
Reynolds number based on boundary layer thickness is shown in figure 35. This is the most relevant Reynolds number for shock - boundary layer interactions because it reflects the ratio of inertial to viscous forces over a length scale consistent with the fact that $\partial \mathrm{u} / \partial \ell \approx \partial \mathrm{u} / \partial \mathrm{h}$ when a shock meets a boundary layer (thereby violating the classic boundary layer assumption that $\partial u / \partial \ell<<\partial u / \partial h)$. As shown, the computation gives $\operatorname{Re}_{\delta}$ values of about 6000-8000 upstream of the shock boundary layer interaction region, typical for a fully developed turbulent boundary layer in a sub-scale CD nozzle.

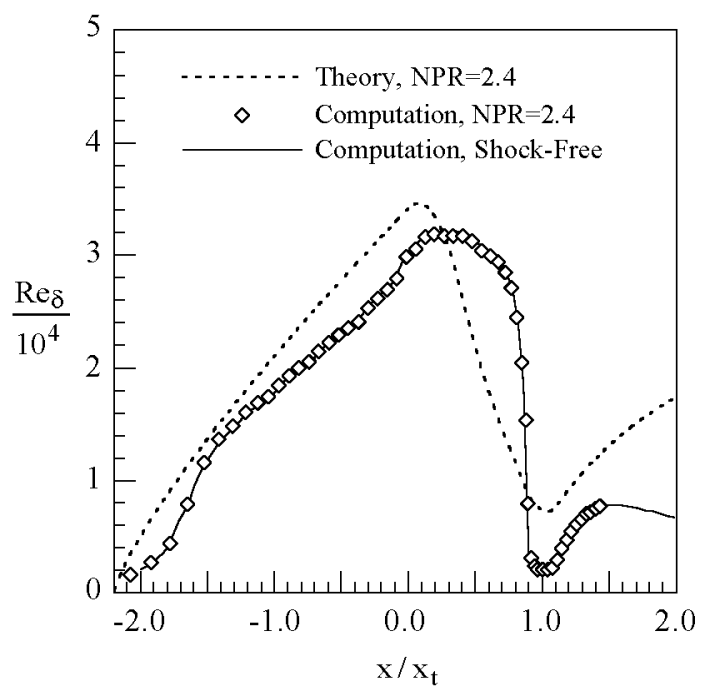

Figure 35: Reynolds Number Based on $\delta$

The boundary layer shape factor $\delta^{*} / \theta$ is presented in figure 36 , and shows that the computed boundary layer was near its theoretical equilibrium throughout most of the convergent section of the nozzle, interrupted slightly by the vena contracta effect at the throat. Downstream, the computed shape factor is seen to fall onto the theoretical curve in the divergent section of the nozzle, indicating that the boundary layer was fully developed and well behaved in this region. This is confirmed by the computational velocity profile at $\mathrm{x} / \mathrm{x}_{\mathrm{t}}=1.4$, presented in wall variables in figure 37 . This profile follows the classic "law of the wall" in the nearwall laminar sublayer for $\mathrm{h}^{+} \leq 5$, goes through a buffer region around $\mathbf{h}^{+}=10$, and then falls onto a logarithmic profile in the inertial sublayer from $50 \leq \mathrm{h}^{+} \leq \mathbf{8 0 0}$ before entering the core region at $\mathrm{h}^{+} \approx 1000$. 


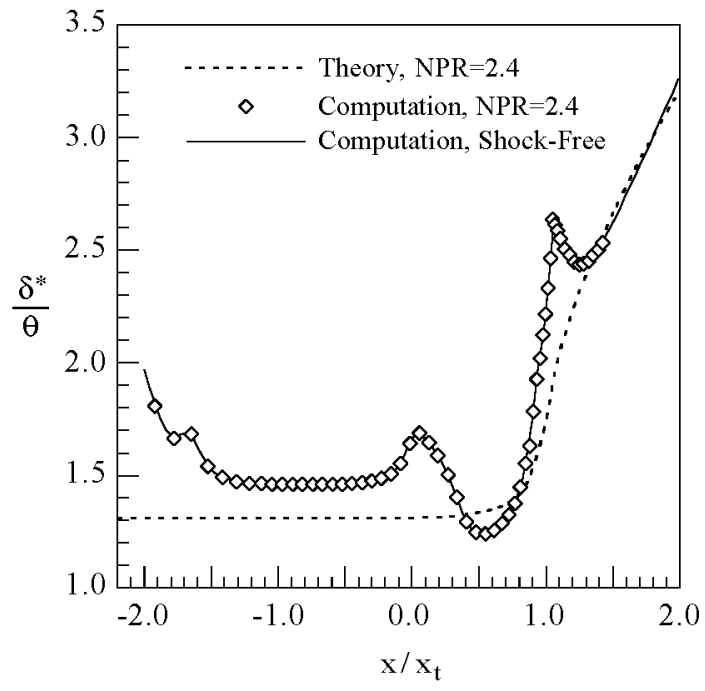

Figure 36: Boundary Layer Shape Factor



Figure 37: Boundary Laver Profile at $x / x_{i}=1.4$

\section{Pressure Comparison}

Experimental and computational pressure data in the shock - boundary layer interaction region are shown at $\mathrm{NPR}=2.4$ in figure 38 , plotted against normalized distance from the shock $\left(\mathrm{x}-\mathrm{x}_{\mathrm{s}}\right) / \delta_{\mathrm{o}}\left(\right.$ where $\left.\mathrm{x}_{\mathrm{s}} / \mathrm{x}_{\mathrm{t}} \approx 1.45\right)$ on the lower abscissa, and $\mathrm{x} / \mathrm{x}_{\mathrm{t}}$ on the upper abscissa. As shown previously, there is good agreement between CFD and experiment. Some adjustment is necessary to compare experimental and computational pressure results with theory, since the theoretical model (based on one-dimensional inviscid flow) predicts a shock free nozzle at this NPR. In figure 39, the nondimensional streamwise pressure gradient $\left(\mathrm{x}_{\mathrm{t}} / \mathrm{p}_{\mathrm{oj}}\right)(\partial \mathrm{p} / \partial \mathrm{x})$ is plotted for experimental and computational data at $\mathrm{NPR}=2.4$ and the theoretical prediction at NPR $=1.33$. All three cases correspond to a shock location of $\mathrm{x} / \mathrm{x}_{\mathrm{t}} \approx 1.45$ and an onset Mach number of $M_{1} \approx 1.6$. Plotting pressure data in terms of the gradient eliminates differences in exit back pressure and allows a fair comparison for a fixed shock location and strength. With this convention, negative values of pressure gradient indicate expansion, positive values indicate compression, and zero values indicate no pressure change (in a duct of varying area, this implies separation).

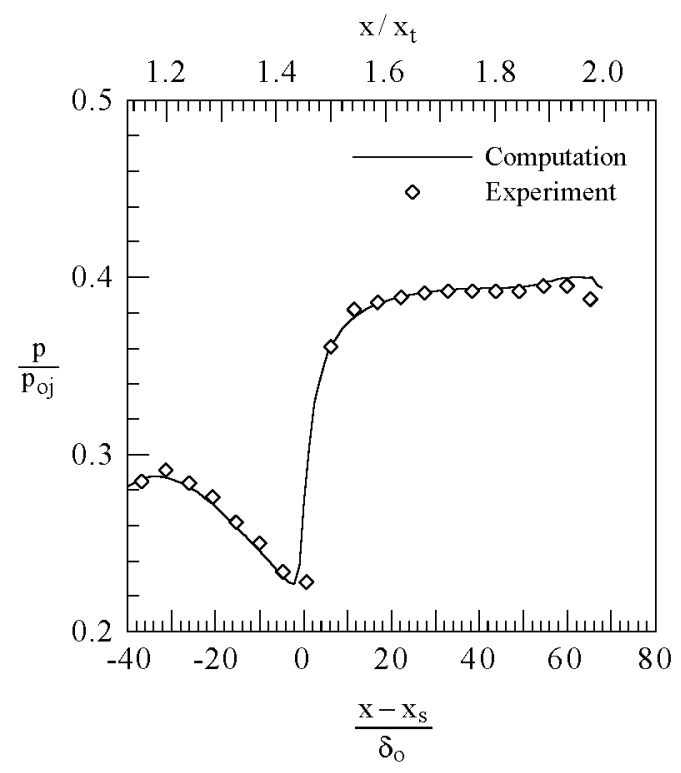

Figure 38: Pressures at NPR $=2.4$

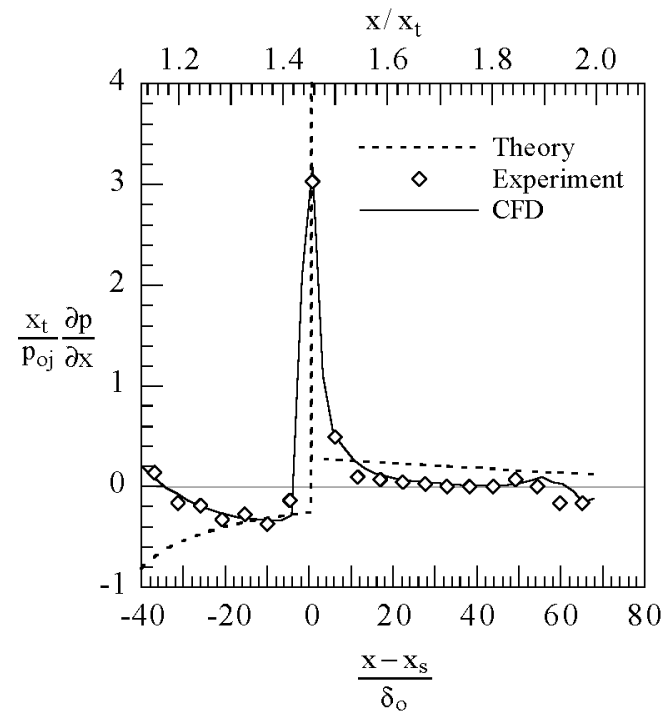

Figure 39: Nondimensional Pressure Gradient 
In the expansion region upstream of the shock, experimental and computational pressure gradient data show rough agreement with theory, though the latter clearly does not account for the vena contracta effect at the nozzle throat. Data in the shock region indicate a single, sharp compression, but one that is gradual and spread out compared to the inviscid theory. This is expected, as viscosity results in a thicker shock and allows for upstream pressure communication through the subsonic portion of the boundary layer. Measured from the point of initial pressure jump to the downstream location where the experimental and computational pressure gradient matched theory, the "pressure length" of the shock - boundary layer interaction was about $16 \delta_{0}$. Whereas theory predicts some pressure recovery downstream of the interaction, experimental and computational data approach a near zero value, representative of full separation.

\section{Computational Boundary Layer Profiles}

Normalized boundary layer velocity profiles from the computation at various streamwise locations through the shock - boundary layer interaction are shown in figure 40 . The first profile at $\mathrm{x}-\mathrm{x}_{\mathrm{s}} / \delta_{\mathrm{o}}=-6.31\left(\mathrm{x} / \mathrm{x}_{\mathrm{t}}=1.4\right)$ is representative of a classic turbulent boundary layer and was also observed at upstream locations. It was the last profile seemingly unaffected by the shock boundary layer interaction, and thus marks the upstream extent of that interaction and the upstream influence of the shock jump (through the subsonic portion of the boundary layer). Moving downstream, boundary layer profiles show influence of the adverse pressure gradient imposed by the shock, and reach an inflectional state at $\mathrm{x}-\mathrm{x}_{\mathrm{s}} / \delta_{\mathrm{o}} \approx 3$. Separation and reverse flow are seen to occur for all subsequent downstream profiles.

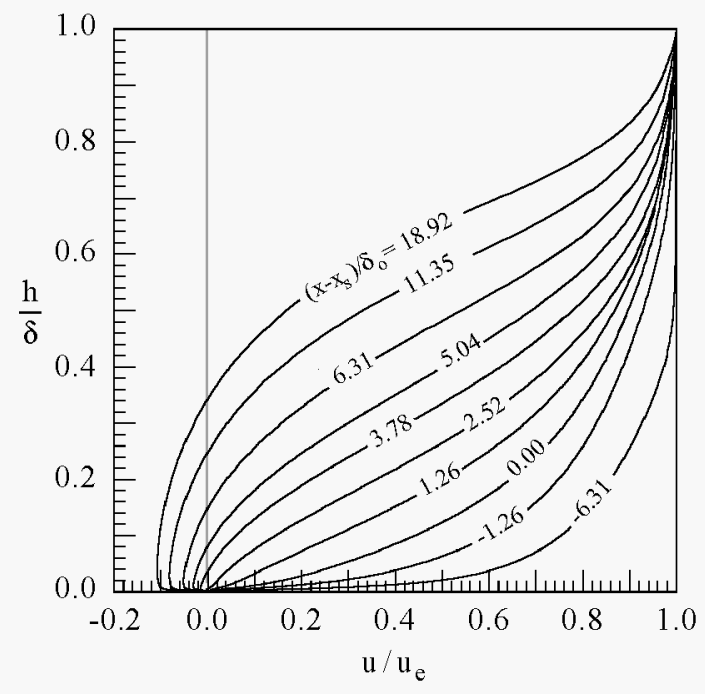

Figure 40: Velocity Boundary Layer Profiles
Combined with previous observations, the boundary layer profiles indicate that, first, the distance between the undisturbed boundary layer at $\mathrm{x}-\mathrm{x}_{\mathrm{s}} / \delta_{\mathrm{o}}=-6.31$ and the initial shock jump (in the computational data) at about $\mathrm{x}-\mathrm{x}_{\mathrm{s}} / \delta_{\mathrm{o}}=-4.9$ was less than $2 \delta_{\mathrm{o}}$. The distance from the shock jump to separation at $\mathrm{x}-\mathrm{x}_{s} / \delta_{0} \approx 3$ was about $8 \delta_{0}$. So, from the standpoint of viscous flow in the nozzle boundary layer, the entire shock - boundary layer interaction and resulting separation occurred over a total distance of about $9-10 \delta_{0}$.

\section{Schlieren Images}

Experimental and computational schlieren flow visualization is shown in figure 41 . Though there are slight differences in shock structure, both images show the nozzle shock with a large, well defined lambda foot, and separation that begins at the leading lambda shock and extends downstream. Coupled with pressure data discussed above, this suggests that as far as the nozzle boundary layer was concerned, all of the pressure rise across the lambda shock system occurred across the leading branch of the lambda foot. In this shock system, the leading branch of the lambda foot extended down to the nozzle boundary layer, while the trailing lambda foot extended to the detached shear layer. In the computational image, vortex sheets are visible downstream of the lambda bifurcation points, and provide an entropy slip between flow that passed through the oblique lambda shocks and flow that passed through the single normal shock. These were also observed in video taken during the experiment.

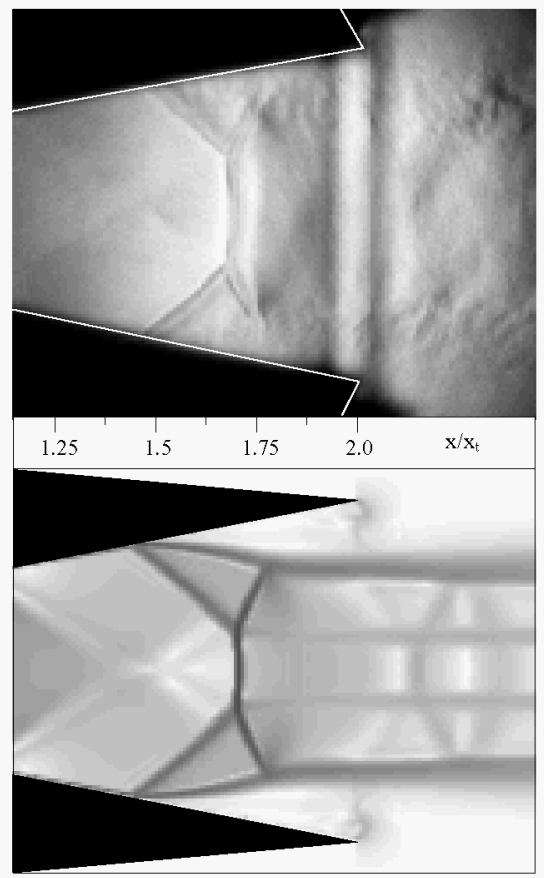

Figure 41: Comparison of Experimental (top) and Computational (bottom) Schlieren Images at NPR $=2.4$ 


\section{Experimental Shock Measurements}

Shock measurements were made from the experimental schlieren image and used in conjunction with pressure data and oblique shock relations to diagram the shock boundary layer interaction. As shown in figure 42, the leading lambda shock had a flow inclination angle $\beta \approx 52^{\circ}$ from the nozzle flap, which, with an upstream Mach number of $M_{1} \approx 1.6$, resulted in a calculated turning angle of $11^{\circ}$ and a downstream Mach number of approximately 1.2 . From this new direction, the trailing shock had an inclination of $\beta \approx 66^{\circ}$, and with $M_{1} \approx 1.2$, the corresponding turning angle was $4^{\circ}$. Overall, this indicates that flow exited the lambda shock system at an angle of $\alpha \approx 4^{\circ}$, or $7^{\circ}$ off of the divergent flap.

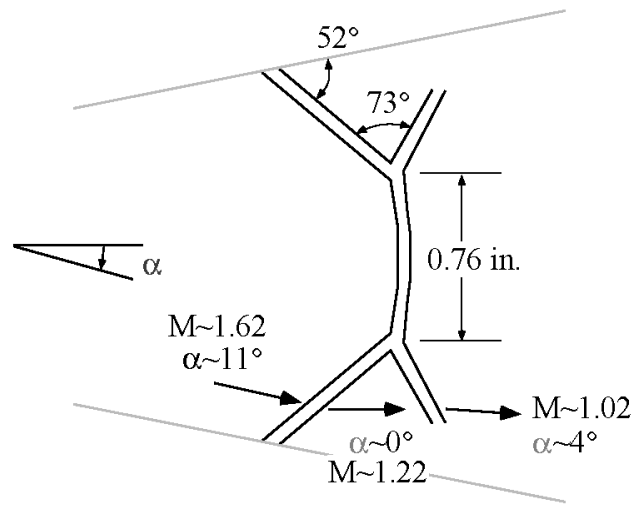

Figure 42: Experimental Shock Schematic

\section{Computational Shock Measurements}

Computational Mach number contours are presented in figure 43 , and streamlines are presented in figure 44 (note the presence of a double eddy recirculation region downstream of the flow separation point which supports earlier discussion). Together, these plots were used to piece together the computational shock schematic shown in figure 45 .

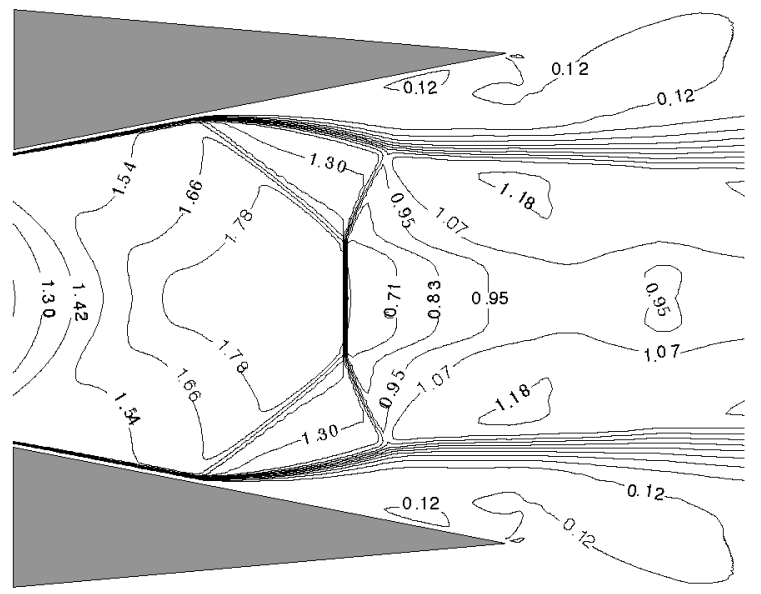

Figure 43: Computational Mach Contours



Figure 44: Computational Streamlines

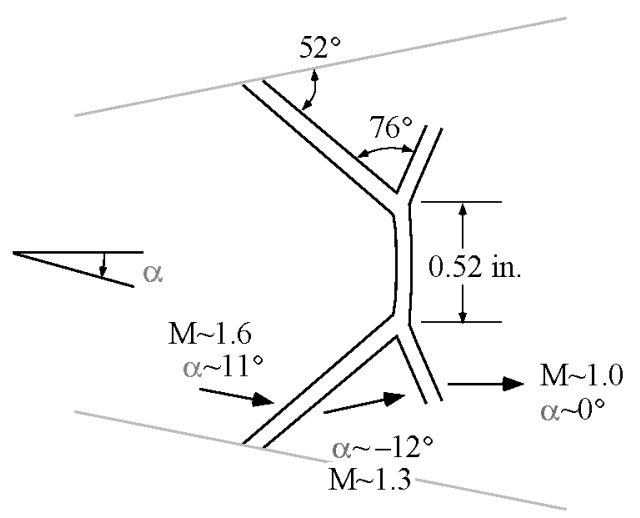

Figure 45: Computational Shock Schematic

For comparison, the experimental and computational shock schematics are overlaid in figure 46. Although the basic lambda foot angles are similar in the experimental and computational schematics, the computational lambda shock structure extends further downstream. Among other things, this shortens the height of the inviscid normal shock and pushes it slightly downstream.

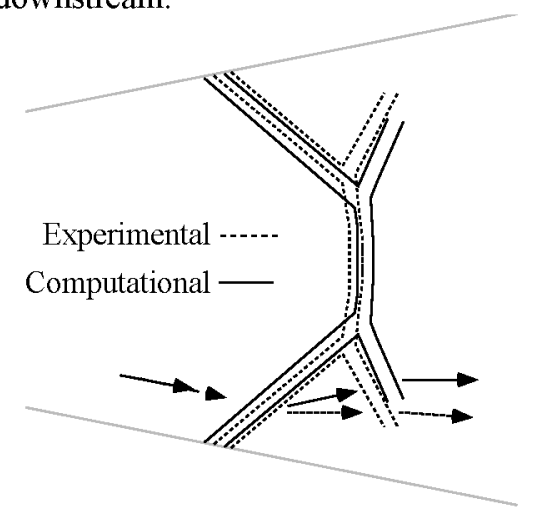

Figure 46: Shock Structure Comparison 
A much bigger difference in the experimental and computational shock structures is evident when comparing flow angles (Mach numbers are in relatively good agreement). After the first lambda shock, the computation shows over twice as much turning as the experimental estimate, and flow exited the lambda shock system in the axial direction, compared to the $4^{\circ}$ angle estimated from the experiment. Part of this discrepancy could be due to the questionable use of oblique shock theory to explore the experimental shock - boundary layer interaction, but this should be a reasonably sound approach, if not totally accurate. Coupled with the differences in shock structure, this flow angle discrepancy indicates that the computationally simulated shock - boundary layer interaction had different turning requirements than the experimental case (possibly due to differences in the complicated double eddy recirculation region). The fact that experimental and computational thrust efficiency trends were similar indicates that the overall "thermodynamic" effect of the shock system was probably the same in both cases, as it should be, since basic shock jump requirements are very simple and "global" in nature.

\section{Concluding Remarks}

An investigation of separated nozzle flows has been conducted using experimental, theoretical, and computational approaches. Specific conclusions are as follows:

1. Experimental results indicate that the test nozzle was dominated by shock induced boundary layer separation at overexpanded off-design conditions. This separation had two distinct regimes: for NPR $\leq 1.8$, the separation was three-dimensional, somewhat unsteady, and confined to a bubble (with partial reattachment over the nozzle flap). For NPR $\geq 2.0$, separation was steady and fully detached, and it became more two-dimensional as NPR increased. Upon increasing NPR from 1.8 to 2.0, the nozzle went through a dramatic transition, dividing the two separated flow regimes. In all cases, separation improved static thrust efficiency when compared to the ideal (attached flow) theoretical model. As the nozzle became shock free, thrust efficiency followed along the theoretical prediction and matched the theoretical peak thrust efficiency

2. The transition from partial separation to fully detached separation observed when NPR was increased from 1.8 to 2.0 resulted in several important observations. With this NPR increase, nozzle flow adjusted to exit back pressure by completely detaching downstream of the shock; conditions up to the shock were nearly the same as the previous NPR. So, this transition was not the result of markedly different onset conditions or a stronger shock - boundary layer interaction, but instead came about through the natural tendency of an overexpanded nozzle flow to detach and reach a more efficient thermodynamic balance. This suggests that in nozzle flows, shock - boundary layer interaction and separation are mutually dependent results, in contrast to the typical view that shock boundary layer interaction is the "cause" of separation.

3. For engineering purposes, the computational simulation was generally in excellent agreement with experimental data. There were notable exceptions at low off-design NPRs, where the 2D computational model was inconsistent with 3D flow observed in the experiment. At these low NPRs, the computation predicted fully detached separation, and completely missed the transition discussed above. For the simulation of flow control schemes that involve separation (either its alleviation or encouragement), these modeling limitations would be unacceptable. This indicates that follow on studies with a 3D computational model are necessary to explore this regime and to study nozzle separation in general. Though the computation accurately modeled the thrust efficiency trend observed in the experiment (with the exception of the low NPR regime), it did not correctly predict the peak thrust efficiency of the nozzle due to the lack of sidewall skin friction effects in the 2D model. A simple estimate was able to account for this discrepancy.

4. Although low NPR results from the computational simulation were undesirable from a modeling standpoint, they were very informative for controlling and improving off-design nozzle performance. By exploring the effect of fully detached separation at each off-design NPR, the computational results illustrated potential benefits of encouraging fully detached separation in an actual nozzle. With this type of control strategy, the entire overexpanded range of nozzle performance would be within $10 \%$ of the peak thrust efficiency for the nozzle geometry tested. This may allow a fixed geometry nozzle to cover an entire flight mission more efficiently than a mechanical system due to reductions in weight and complexity.

5. From a scientific point of view, the computation was unable to precisely match the shock - boundary layer interaction structure observed in the experiment at an NPR where flow was predominantly 2D. Overall, this had no apparent effect on the nozzle performance trend, but it could be important for studies involving flow control and thrust vectoring, where shock structure and small scale flow features are important. Combined with the need for 3D modeling at low NPRs, this indicates that further work needs to be done (possibly involving grid and solver refinement) to better model the shock boundary layer interaction and shock induced boundary layer separation characteristics in off-design nozzles. 


\section{Acknowledgments}

Part of this research was conducted while the author was a graduate student with the George Washington University Joint Institute for Advancement of Flight Sciences at NASA Langley Research Center. The faculty and staff of JIAFS are thanked for their contributions, and the NASA Langley Configuration Aerodynamics Branch is thanked for supporting this work under NASA grant NCC1-24.

\section{References}

1. Lewis, W.G.E. "Propelling Nozzle Research". Journal of the Royal Aeronautical Society, Volume 68, pages 717-727, November 1964.

2. Asbury, S.C., Gunther, C.L., and Hunter, C.A. "A Passive Cavity Concept for Improving the OffDesign Performance of Fixed-Geometry Exhaust Nozzles". AIAA 96-2541, July 1996.

3. Hunter, C.A. "An Experimental Analysis of Passive Shock - Boundary Layer Interaction Control for Improving the Off-Design Performance of Jet Exhaust Nozzles". M.S. Thesis, The George Washington University JIAFS. September 1993.

4. Pack, L.G., and Joslin, R.D. "Overview of Active Flow Control at NASA Langley Research Center". SPIE 5th Annual International Symposium on Smart Structures and Materials, Paper number 3326-22, March 1998.

5. Staff of the Propulsion Aerodynamics Branch. "A User's Guide to the Langley 16-Foot Transonic Tunnel Complex, Revision 1" NASA TM-102750, 1990 .

6. Mercer, C.E., Berrier, B.L., Capone, F.J., and Grayston, A.M. "Data Reduction Formulas for the 16-Foot Transonic Tunnel at NASA Langley Research Center, Revision 2". NASA TM-107646, 1992.

7. Coleman, H.W., and Steele, W.G. Jr. "Experimentation and Uncertainty Analysis for Engineers". John Wiley \& Sons, 1989.

8. Wing, David J., Mills, Charles T.L., and Mason, Mary L. "Static Investigation of a Multiaxis Thrust-Vectoring Nozzle With Variable Internal Contouring Ability". NASA TP-3628, 1997.

9. Weinstein, L.M. "An Improved Large-Field Focusing Schlieren System". AIAA 91-0567, January 1991.
10. Hunter, C.A. "An Approximate Theoretical Method for Modeling the Static Thrust Performance of Non-axisymmetric TwoDimensional Convergent-Divergent Nozzles". NASA CR-195050, March 1995.

11. Pao, S.P., and Abdol-Hamid, K.S. "Numerical Simulation of Jet Aerodynamics Using a Three Dimensional Navier Stokes Method (PAB3D)". NASA TP-3596, September 1996.

12. Abdol-Hamid, K.S. "Implementation of Algebraic Stress Models in a General 3-D Navier-Stokes Method (PAB3D)". NASA CR-4702, December 1995.

13. Abdol-Hamid, K.S., Lakshmanan, B., and Carlson, J.R. "Application of the Navier-Stokes Code PAB3D with $k-\varepsilon$ Turbulence Models to attached and Separated Flows". NASA TP-3480, January 1995.

14. Carlson, J.R. "High Reynolds Number Analysis of Flat Plate and Separated Afterbody Flow Using Non-Linear Turbulence Models". AIAA 96-2544, July 1996.

15. Shih, T.H., Zhu, J., and Lumley, J.L. "A New Reynolds Stress Algebraic Equation Model". NASA TM-106644, August 1994.

16. Liepmann, H.W., and Roshko, A. "Elements of Gasdynamics". John Wiley \& Sons, 1957.

17. Capone, F.J., Konarski, M., Stevens, H.L., and Willard, C.M. "Static Performance of Vectoring/Reversing Non-axisymmetric Nozzles". AIAA 77-840, July 1977.

18. Leavitt, L.D., and Re, R.J. "Static Internal Performance Including Thrust Vectoring and Reversing of Two-Dimensional ConvergentDivergent Nozzles". NASA TP-2253, 1984.

19. Gatski, T.B., and Speziale, C.G. "On Explicit Algebraic Stress Models for Complex Turbulent Flows". NASA CR-189725, November 1992.

20. Girimaji, S.S. "Fully-Explicit and Self-Consistent Algebraic Reynolds Stress Model". ICASE 95-82, December 1995.

21. Reshotko, E., and Tucker, M. "Effect of a Discontinuity on Turbulent Boundary Layer Thickness Parameters with Application to ShockInduced Separation". NASA TN-3454, 1955. 